\title{
La pensée des enseignants pendant l'interaction en classe. Une revue de la littérature anglophone
}

Teacher thinking during classroom interaction. A review of the "English language" literature

Philippe Wanlin et Marcel Crahay

\section{OpenEdition}

\section{Journals}

Édition électronique

URL : http://journals.openedition.org/educationdidactique/1287

DOI : 10.4000/educationdidactique.1287

ISSN : $2111-4838$

\section{Éditeur}

Presses universitaires de Rennes

\section{Édition imprimée}

Date de publication : 31 mai 2012

Pagination : $9-46$

ISBN : 978-2-7535-1872-8

ISSN : 1956-3485

\section{Référence électronique}

Philippe Wanlin et Marcel Crahay, « La pensée des enseignants pendant l'interaction en classe. Une revue de la littérature anglophone », Éducation et didactique [En ligne], 6-1 | 2012, mis en ligne le 30 avril 2014, consulté le 08 décembre 2020. URL : http://journals.openedition.org/educationdidactique/1287 ; DOI : https://doi.org/10.4000/educationdidactique.1287 


\title{
LA PENSÉE DES ENSEIGNANTS PENDANT LINTERACTION EN CLASSE \\ UNE REVUE DE LA LITTÉRATURE ANGLOPHONE
}

\author{
Philippe Wanlin (Université de Génève) \\ Marcel Crahay (Université de Génève et de Liège)
}

\begin{abstract}
Résumé : Ce texte se penche sur la manière dont les chercheurs ont schématisé la pensée interactive des enseignants, sur les éléments auxquels ils pensent quand ils donnent leurs cours et sur les facteurs qui influencent cette réflexion. Létude de ces éléments de réflexion et des facteurs qui les influencent aboutit quasi inévitablement sur la notion de dilemme qui devient le noyau dur des processus de pensée des enseignants lorsqu'ils enseignent. Ce texte débouche notamment sur une typologie des dilemmes auxquels les enseignants sont confrontés lors de l'interaction en classe. Enfin, il présente une proposition d'organisation des constats de recherche du paradigme du teacher thinking pouvant être considéré comme une heuristique permettant de structurer cette littérature.
\end{abstract}

Mots clés : enseignant, interaction, classe, croyances et connaissances, réflexion

Philippe Wanlin \& Marcel Crahay

En 1986, Clark et Peterson proposent un modèle pour présenter les axes de recherches du paradigme du teacher thinking en les mettant en relation avec les recherches processus-produit. La partie de ce modèle relative aux processus cognitifs des enseignants est composée de trois pôles et intègre une composante transversale, le jugement de la valeur scolaire des élèves par les enseignants. Ces trois pôles sont: la pensée des enseignants durant la planification, la pensée des enseignants durant l'interaction en classe et, le bagage des croyances et connaissances des enseignants. La revue de question que nous proposons ici se penche sur les recherches qui se sont intéressées à la pensée interactive des enseignants ${ }^{1}$. Nous pensons qu'il est important de tenter actuellement une synthèse de ces recherches car nous sommes - pensons-nous - à un tournant du paradigme du teacher thinking: les recherches de la dernière décennie s'intéressent surtout au répertoire cognitif des enseignants et, parfois, à la concordance entre les croyances ou connaissances des enseignants et leur pratique d'enseignement.

Les recherches qui se sont intéressées à la pensée enseignante durant l'interaction en classe se sont organisées autour de deux grands thèmes: 1) la modélisation de la réflexion interactive et 2) la description du contenu et des influences de cette réflexion. Ce texte est structuré selon ces deux axes de recherches du teacher thinking.
Après un rappel concernant les méthodes utilisées par les chercheurs pour étudier les pensées des enseignants en cours d'enseignement, nous discutons, dans une deuxième partie, la manière dont les chercheurs ont défini la décision interactive. Nous passons ensuite en revue les études qui se sont intéressées à la fréquence et à l'ampleur des décisions interactives des enseignants ainsi qu'à leurs répercussions sur leur plan d'activité et leurs comportements en classe. Dans la foulée, nous examinons de façon critique les diverses tentatives de schématiser le processus de prise de décisions interactives des enseignants et proposons quelques pistes d'amélioration.

Dans une troisième partie, nous nous intéressons aux contenus de la pensée interactive (les préoccupations) et sur les éléments que les enseignants prennent en compte pour penser leurs interventions pédagogiques (les facteurs d'influence). Nous y proposons une typologie des préoccupations interactives des enseignants et des indices qu'ils prennent en compte pour moduler leurs réflexions. Ceci aboutit à la notion de dilemme: la prise en considération des multiples dimensions de la réalité enseignante confronte les maîtres à des choix quasi insolubles (par exemple, décider d'avancer avec les forts ou de se concentrer sur les plus faibles). Les tensions liées à des perspectives perçues comme inconciliables sont difficiles à gérer car toute décision prise dans un sens entraîne des effets non désirés. Dans une quatrième 
partie, nous nous pencherons donc sur les dilemmes vécus par les enseignants, tenterons d'en proposer une typologie, mais aussi examinerons les procédures compensatoires mises en place par ceux-ci pour minimiser les effets non désirés des choix posés en un premier temps.

Enfin, cette revue de la littérature se conclut par un modèle théorique de la réflexion interactive des enseignants. Ce modèle, non validé empiriquement, a une visée heuristique: tout en offrant une illustration du fonctionnement possible de la pensée interactive, il permet d'organiser les constats des recherches du teacher thinking. Une courte discussion conclut ce texte en situant les apports du teacher thinking pour la formation initiale et continue des enseignants ainsi qu'en ouvrant de nouvelles perspectives pour la recherche en sciences de l'éducation.

Comme indiqué ci-dessus, en entrée en matière dans le champ de recherche passé ici en revue, il est indispensable de discuter sur le plan méthodologique les deux manières les plus utilisées par les chercheurs pour étudier la pensée interactive.

\section{Comment la pensée interactive a été étudiée?}

Bien que diverses méthodes aient été utilisées pour étudier la pensée interactive des enseignants, le relevé de la littérature fait apparaître deux techniques privilégiées: le stimulated recall ou rappel stimulé et la policy capturing ${ }^{2}$. Bon nombre des recherches que nous présentons ici utilisent une variante de l'une ou de l'autre de ces méthodes de recherche.

\section{Rappel stimulé: commentaire de son enseignement}

Le rappel stimulé consiste à présenter une bande vidéo ou audio d'un épisode d'enseignement afin de permettre à l'enseignant protagoniste de cet épisode de rapporter, lors d'un entretien, ses pensées et ses prises de décisions durant l'épisode d'enseignement dont il est question (Borko \& Shavelson, 1990; Clark $\&$ Peterson, 1986; Clark \& Yinger, 1978; Shavelson \& Stern, 1981; Tochon, 1993a). Généralement, ces interviews enregistrées sont transcrites et leur contenu est codé par catégories donnant lieu à des comptages d'unités de réflexion (Borko \& Shavelson, 1990; Clark \& Peterson, 1986).

Diverses études ont utilisé cette technique (e.a.: Morine-Dershimer, 1979; Smith \& Sendelbach, 1979; McLeod, 1981; Calderhead, 2003; Tillema, 2003). Par exemple, Wanlin (2009b, 2009c) filme des enseignants du primaire et leur demande de commenter leur enseignement, d'abord de manière libre et, ensuite, en utilisant un canevas de questions standards lors d'interviews semi dirigés. À noter que cette technique, bien qu'utilisée souvent avec une observation filmée de l'enseignement en classe, a souvent été utilisée sans observation filmée, mais au départ d'une retranscription effectuée par le chercheur. De plus, rares sont les chercheurs qui ont exploité les données issues des observations et les ont mises en relation avec les propos des enseignants. Or, les données recueillies par le biais du rappel stimulé reflètent probablement un amalgame de souvenirs véridiques des processus cognitifs durant l'interaction d'enseignement et des éléments issus du traitement d'informations par l'enseignant lors $\mathrm{du}$ visionnement de son enseignement (Peterson $\&$ Clark, 1978). Enfin, il convient de signaler que, dans de nombreuses recherches, le rappel stimulé est combiné à d'autres méthodes de recueil de données: questionnaires, échelles de jugements, etc.

\section{Policy capturing: questionnaires ou échelles sur les « politiques " de décision et d'évaluation des enseignants}

La policy capturing, empruntée à la psychologie expérimentale de laboratoire, consiste à présenter, à des enseignants, une série de descriptions d'élèves, de situations d'enseignement ou de matériel curriculaire et de les inviter à porter un jugement à leurs propos (Clark \& Peterson, 1986). Ces descriptions, rédigées par les chercheurs, font varier plusieurs indices afin d'en évaluer l'importance. Les jugements des enseignants sont généralement enregistrés sur une échelle de Likert et les données servent communément à la production de modèles statistiques permettant d'analyser le poids des différents paramètres intégrés dans les descriptions soumises aux enseignants.

L'une des recherches les plus représentatives de l'utilisation de cette méthodologie est vraisem- 
blablement celle de Borko et Cadwell (1982). Ces deux chercheurs ont tenté d'analyser les jugements des enseignants sur la valeur scolaire de leurs élèves (compétences académiques, motivation et comportement en classe) et leurs décisions par rapport à l'organisation et la gestion de la classe ou des contenus. Pour ce faire, ils ont présenté à 4 enseignants du degré supérieur de l'enseignement primaire, ayant entre 1 et 13 ans d'expérience dans ce niveau, des descriptifs d'élèves variant sur six facteurs: le sexe, le niveau de performance antérieur, les conduites en matière de respect des règles, l'autonomie, les compétences sociales et la confiance en soi. Ces six facteurs ont été retenus car ils représentent le type d'informations auquel les enseignants recourent pour former leurs impressions vis-à-vis de leurs élèves ( $c f$. Borko, Cone, Atwood-Russo, \& Shavelson, 1979). De surcroît, ces informations sont disponibles dès les premières semaines de l'année scolaire. Parallèlement, les enseignants sont invités à répondre à un questionnaire permettant, d'une part, d'analyser si leurs pratiques instructionnelles se rapprochent davantage d'un enseignement traditionnel ou non et, d'autre part, d'identifier quelques variables caractérisant leur expérience d'enseignement, leur participation à des programmes spéciaux, le type d'école, le niveau d'études, la classe où ils interviennent, etc.

Cette technique a parfois été utilisée pour des investigations à plus large échelle (Cone, 1978) et, dans certaines études (Shavelson, Atwood, \& Borko, 1977) pour comparer des enseignants à des profanes. Elle a aussi été déclinée de diverses manières: ainsi, Natriello et Dornbusch (1983) ont demandé à des enseignants de décrire certains de leurs élèves réels plutôt que de les confronter à des portraits d'élèves fictifs. À nouveau, on peut remarquer dans la littérature que la policy capturing a souvent été accompagnée de diverses variantes de questionnaires ou d'interviews.

\section{Comment la pensée interactive a été modélisée par les chercheurs}

Cette partie est composée de trois sections: la définition du concept de décision interactive, la description de modèles décisionnels et une critique de ces tentatives de schématiser les prises de décision des enseignants en cours d'interaction avec les élèves.

\section{Décision interactive: définition du concept et estimation de la fréquence des prises de décision}

Les recherches dans le domaine ont pour point de départ principal un article de Shavelson (1973), qui y formule l'idée que tout acte d'enseignement est le résultat d'une décision consciente ou inconsciente que l'enseignant prend après un processus de traitement cognitif complexe d'informations disponibles. Ce raisonnement mène ce chercheur à faire l'hypothèse que la compétence de base en enseignement est la capacité de prendre des décisions. Les problèmes méthodologiques évidents sousjacents à la description des décisions inconscientes ont poussé les chercheurs à abandonner cette partie du raisonnement de Shavelson pour se concentrer sur les décisions conscientes.

Les premières définitions de la notion de décision interactive incluaient la nécessité d'alternatives - plusieurs pour Marland (1977) ou au moins deux pour Sutcliffe et Whitfield (1979) - et la modification ou non du comportement enseignant (Fogarty, Wang, \& Creek, 1982; Marland, 1977; Morine \& Vallance, 1975; Shroyer, 1981; Sutcliffe \& Whitfield, 1979; Wodlinger, 1980). Les recherches ayant trait à la prise en considération de cours d'action alternatifs ont abouti à des résultats mitigés. Ainsi, pour Morine et Vallance (1975), les enseignants en considèrent en moyenne 3 alors que, pour Marland (1977), c'est 2 et que, pour Wodlinger (1980), ce ne serait qu'un seul. Néanmoins, les travaux de recherche ont rapidement montré que les enseignants décidaient sans forcément recourir à un grand nombre d'alternatives (Marland, 1977; Shroyer, 1981). Tous les chercheurs qui ont tenté de préciser la notion de décisions interactive se sont donc, en quelque sorte, accordés pour la définir comme étant un choix délibéré d'implémenter une action spécifique, à savoir de modifier ou non son comportement (Borko \& Shavelson, 1990; Clark \& Peterson, 1986).

Une fois la notion de décision interactive définie, les chercheurs ont tenté d'en mesurer la fréquence. Ces recherches ont obtenu des résultats consistants malgré l'utilisation de méthodes différentes. Les enseignants prennent en moyenne entre 0,5 et 0,7 décisions par minute d'interaction (Fogarty et al., 1982; Marland, 1977; Morine \& Vallance, 1975; Shroyer, 1981; Wodlinger, 1980). Ceci signifie qu'en 
moyenne, les enseignants prennent une décision interactive environ toutes les deux minutes (Borko \& Shavelson, 1990; Clark \& Peterson, 1986; Dunkin, 1986). Bref, les demandes en prise de décision interactives en situation d'enseignement sont relativement intenses (Clark \& Peterson, 1986³).

L'importance des décisions interactives démontrée empiriquement, les chercheurs se sont logiquement interrogés sur la nature du processus décisionnel et ont tenté de modéliser ce qu'ils estimaient être une compétence de base pour enseigner.

\section{Schématisation des processus de prise de décision des enseignants lors des interactions en classe}

De Snow à Peterson et Clark: impacts des comportements d'élèves sur les comportements des enseignants

Snow (1972) a décrit les processus de pensée interactive comme étant un processus cyclique d'observation du comportement des élèves suivi d'un jugement portant sur leur adéquation au déroulement de l'activité, la question étant d'estimer si leurs comportements sont dans des limites désirables. Ce jugement est suivi, à son tour, par une décision de continuer le processus d'enseignement de manière inchangée ou de chercher en mémoire un comportement d'enseignement alternatif qui pourrait ramener le comportement des élèves dans les limites de tolérance. Dans le cas où aucune alternative n'est disponible en mémoire, l'enseignant continue son enseignement de manière inchangée; si une alternative plausible est disponible, l'enseignant peut décider d'agir sur la base de cette alternative en changeant le cours de l'instruction, ou peut l'ignorer et continuer son enseignement de manière inchangée. La figure 1 illustre cette séquence d'événements et le tableau 1 présente les quatre trajectoires possibles de ce modèle.

Pour Snow (1972), l'indice sur lequel se base l'enseignant lors de ses prises de décisions interactives est le comportement des élèves en classe. Selon Peterson et Clark (1978), cet indice comprend la compréhension de la leçon et une participation appropriée de la part des élèves.

\begin{tabular}{|l|c|c|c|c|}
\hline \multicolumn{1}{|c|}{ Carrefours décisionnels } & Trajectoire 1 & Trajectoire 2 & Trajectoire 3 & Trajectoire 4 \\
\hline $\begin{array}{l}\text { Participation des élèves dans les marges } \\
\text { de tolérance }\end{array}$ & OUI & NON & NON & NON \\
\hline Existence d'actions alternatives possibles & & NON & OUI & OUI \\
\hline $\begin{array}{l}\text { Décision d'implémenter une alternative } \\
\text { d'enseignement }\end{array}$ & & & NON & OUI \\
\hline
\end{tabular}

Tableau 1: Trajectoires décisionnelles et comportementales du modèle de Snow (1972) ainsi que de Peterson et Clark (1978)

Peterson et Clark (1978) ont vérifié la pertinence du modèle qu'ils ont conçu à partir de l'apport de Snow (1972) ${ }^{4}$. Ils ont payé 12 enseignants (6 hommes et 6 femmes) expérimentés (8,5 années d'ancienneté en moyenne) et 288 élèves. Trente-six groupes de 8 étudiants ont été constitués de façon aléatoire, après une stratification de l'échantillon d'élèves sur la base du genre et des aptitudes verbales. Chaque enseignant a enseigné à trois groupes différents une séquence de trois leçons (de 50 minutes) portant sur une thématique de social studies, et ceci au cours de la même journée. Le matin, les ensei- gnants disposaient de 90 minutes pour planifier la séquence de leçons. Ensuite, ils enseignaient les leçons préparées; celles-ci étaient filmées. Au terme des trois leçons, les étudiants répondaient à un test à choix multiples, rédigeaient un essai (essay test) et remplissaient un questionnaire d'attitude. Après chaque leçon, les enseignants visionnaient quatre extraits du film de leur enseignement et répondaient aux questions d'une interview structurée relative à leurs processus cognitifs durant l'enseignement. Ces interviews étaient transcrites puis codées pour identifier des séquences correspondant aux définitions des 
carrefours décisionnels (voir tableau 1). Les résultats montrent qu'en règle générale, l'enseignant juge que le comportement des élèves en classe se situe à l'intérieur des limites de tolérance (trajectoire 1). C'est la trajectoire la plus souvent citée (de 70 à $60 \%$ ) par les enseignants. Pour Peterson et Clark (1978), ceci s'explique de plusieurs manières. La majorité de l'interaction en classe peut être décrite au mieux comme étant du «business as usual » (Goodlad \& Klein, 1970; Hoetker \& Ahlbrand, 1969) et, les enseignants conduisent les activités de façon routinière (Dunkin, 1986). Ils tolèrent probablement les comportements des élèves dans des limites à amplitude plus élevée qu'on ne le pense ou bien les comportements des élèves étaient, lors de l'étude en laboratoire de Peterson et Clark, dans des limites tolérables.

Il paraît fort probable que les enseignants agissent lors de l'interaction pour maintenir les comportements des élèves dans des limites de tolérance acceptables à leurs yeux. Ainsi, les résultats de Parker et
Gehrke (1986, 1984) montrent que les enseignants appliquent certaines procédures comportementales visant à assurer la complétion du plan mental de l'activité, plan qu'ils mémorisent lors de la planification et qu'ils maintiennent en tête lors de l'interaction. De plus, leurs constats indiquent que le risque de perte de temps, de non-respect du contenu et de comportements non tolérables de la part des élèves qui accompagne le changement de trajectoire est trop important aux yeux des enseignants qui rechignent à emprunter une autre voie que celle qu'ils ont privilégiée lors de la planification. De même, les enseignants tendraient à rester fidèles à leur planification probablement parce qu'ils estiment que toute modification risque d'entraîner des conséquences négatives (J. Putnam \& Duffy, 1984). Cette tendance peut cependant parfois s'avérer contreproductive surtout lorsque les besoins des élèves ne sont pas rencontrés (Parker \& Gehrke, 1986). Ceci nous amène donc aux trajectoires suivantes du modèle de Peterson et Clark (1978) (cf. figure 1).

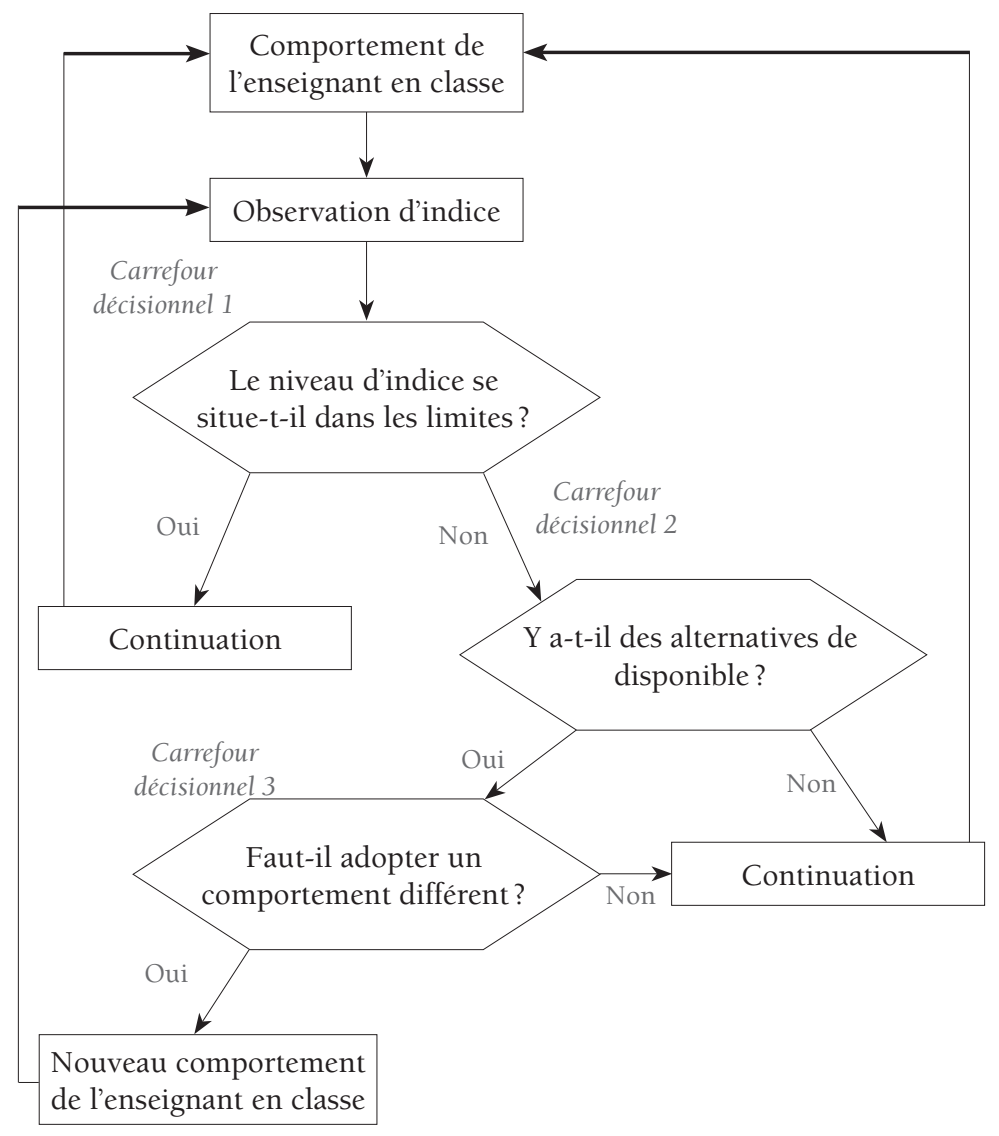

Figure 1: Modèle des processus cognitifs des enseignants durant l'interaction d'après Snow (1972, dans Clark et Peterson, 1986) 


\section{LA PENSÉE DES ENSEIGNANTS PENDANT L'INTERACTION EN CLASSE}

Philippe Wanlin \& Marcel Crahay

Cumulativement, les trajectoires 2, 3 et 4 représentent en moyenne entre 30 et $40 \%$ des stratégies d'enseignement. Dans la trajectoire 2, l'enseignant juge que le comportement des élèves en classe ne se situe pas dans les limites de tolérance acceptables, mais il n'a pas de stratégie disponible dans son répertoire comportemental. Cette trajectoire qui est citée en troisième position traduit, selon Peterson et Clark (1978), un sentiment de surprise et d'impuissance de la part des enseignants. Dans la trajectoire 3, l'enseignant juge que le comportement des élèves en classe ne se situe pas dans des limites de tolérance acceptables et dispose de comportements alternatifs dans son répertoire, mais décide de ne pas changer son enseignement. Cette trajectoire qui est citée la moins souvent par les enseignants de Peterson et Clark (1978) indique que les enseignants estiment que la stratégie planifiée est la meilleure à utiliser parmi un ensemble d'autres stratégies moins bonnes ou plus risquées. Pour les deux chercheurs, si un enseignant rapporte avoir eu une alternative en tête sans l'avoir effectuée, c'est probablement parce qu'il poursuit un objectif instructionnel qu'il a fixé lors de la planification. Les enseignants qui mentionnent le plus cette trajectoire tendent à être moins efficaces: leurs élèves ont des performances moindres et apprécient moins l'enseignant, la méthode et le contenu.

Dans la trajectoire 4, l'enseignant juge que le comportement des élèves en classe ne se situe pas dans des limites de tolérance acceptables, dispose de comportements alternatifs dans son répertoire et décide de se comporter selon cette alternative pour ramener le comportement dans des limites de tolérance acceptables. Cette trajectoire, qui, selon nous, montre une plus grande capacité d'adaptation et une régulation de l'action en cours, est citée en deuxième position par les enseignants (entre 10 et $25 \%$ ).
L'apport de Shavelson et Stern: impact des comportements d'élèves sur l'implémentation de routines d'enseignement

Shavelson et Stern (1981) ont proposé un modèle sur la base d'apports de divers chercheurs (Joyce, 1978; Peterson \& Clark, 1978; Shavelson, 1976; Snow, 1972). Ils partent du postulat que l'enseignement interactif peut être caractérisé comme l'exécution de routines bien établies (Borko \& Shavelson, 1990; Clark \& Peterson, 1986; Shavelson \& Stern, 1981). Selon Shavelson et Stern (1981), les routines minimisent la prise de décision consciente lors de l'enseignement interactif et permettent le maintien du flux d'activités tout en diminuant la charge de traitement des informations et en augmentant la prédictibilité du séquençage des activités, le timing et les comportements des élèves et de l'enseignant lui-même. À noter que l'idée de routine n'est pas de Shavelson et Stern (1981), mais de Yinger (1977), Morine-Dershimer (1978a, 1978b, 1978c) et de Joyce (1978). L'apport de Shavelson et Stern (1981) consiste à poser que la décision interactive intervient quand la routine d'enseignement est interrompue (Borko \& Shavelson, 1990; Clark \& Peterson, 1986); ceci constitue une nuance importante de l'idée initiale de Shavelson qui a manifestement tenu compte des critiques formulées à son article de 1973.

Pour ces deux chercheurs, l'enseignant forme, lors de la planification, une image mentale faisant office de plan d'exécution de l'enseignement interactif. Ces plans ou images sont routinisé(e)s de telle manière qu'une fois entamé(e)s, ils/elles sont généralement exécutés jusqu'à leur terme. À nouveau, d'après Shavelson et Stern (1981), le processus de prise de décisions des enseignants inclut l'observation d'indices (le comportement des élèves) et le jugement consistant à estimer si ces indices se situent dans des limites tolérables (fig. 2). Si le comportement des 
élèves ne se situe pas dans des limites tolérables, l'enseignant décide de la nécessité d'une action immédiate. Le cas échéant, l'enseignant décide (1) d'exécuter une routine alternative s'il y en a une de disponible; (2) de postposer sa réaction si une action différée peut être préférée à une action immédiate; ou (3) de continuer la routine d'enseignement de manière inchangée.

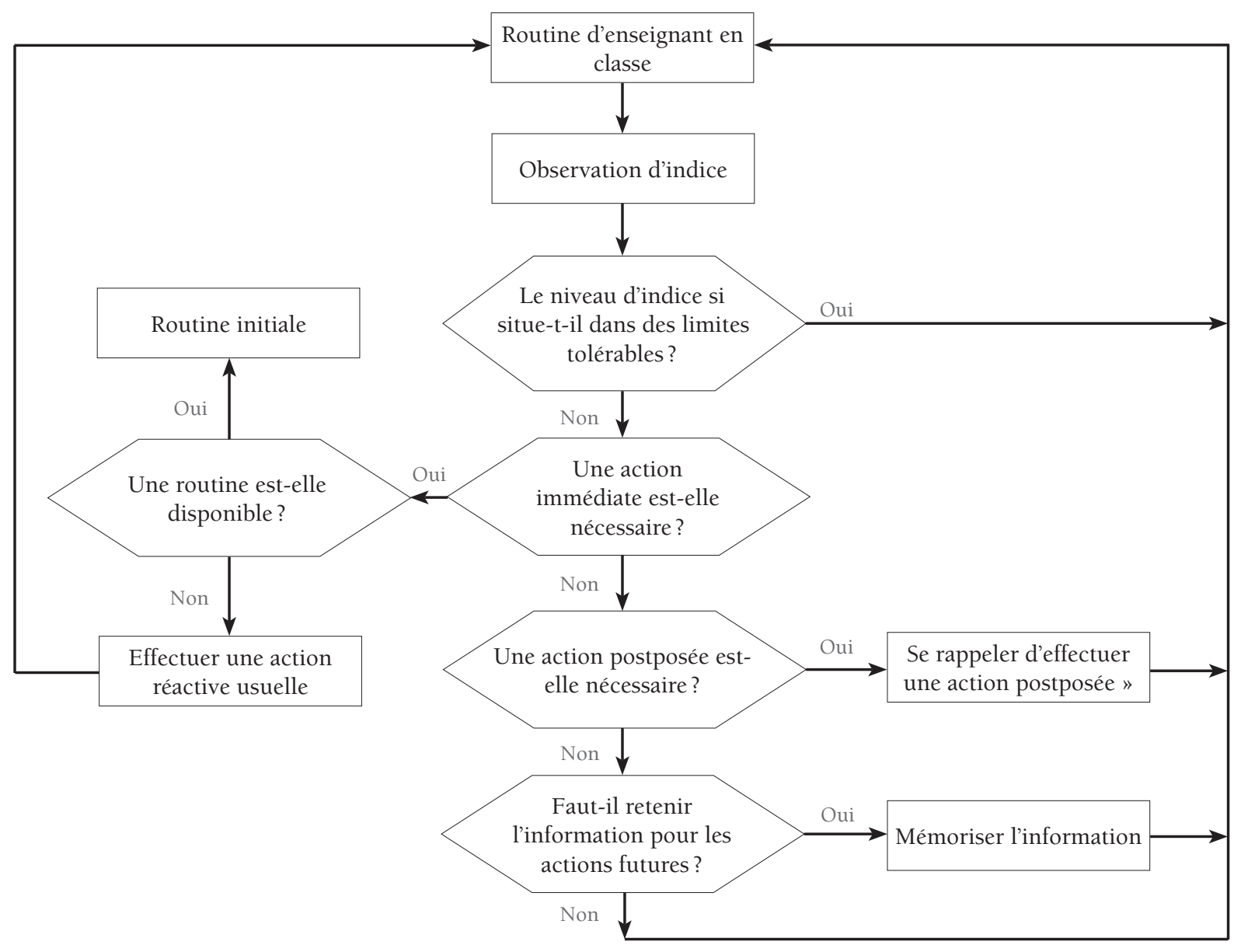

Figure 2: Modèle de la prise de décisions interactives des enseignants (Shavelson E Stern, 1981) 


\section{LA PENSÉE DES ENSEIGNANTS PENDANT L'INTERACTION EN CLASSE}

\section{Philippe Wanlin \& Marcel Crahay}

\section{Modèle de Kleven: de l'observation critique à l'action réfléchie}

Pour Kleven (1991), l'idée séminale de Shavelson (1973) consistant à assimiler l'enseignement à un processus de prises de décision laisse entrevoir un modèle général de sens commun de l'enseignement interactif qui inclut tant des décisions conscientes que des décisions partiellement ou totalement inconscientes. Avec son modèle (fig. 3), Kleven (1991) postule que l'enseignant choisit des actions appropriées à la situation et aux objectifs d'enseignement. Son modèle du processus de prise de décisions interactives prend en compte les caractéristiques de la vie en classe comme étant multidimensionnelles, simultanées, immédiates, imprédictibles,...

Dans le modèle de Kleven (1991), le processus de prise de décision est composé de plusieurs phases. Lors de la phase de perception, les compétences de monitoring de l'enseignant sont très importantes (withitness, overlapping 5 ,...). L'observation d'indices situationnels de la part de l'enseignant l'amène à une phase de jugement. Le modèle sous-entend que le jugement des situations d'enseignement dépend de la capacité de traitement de l'information disponible et que la qualité de ce jugement est fonction de variables cognitives et émotionnelles. Les variables cognitives renferment plusieurs types de connaissances dont les connaissances pratiques, les connaissances des élèves directement ou indirectement impliqués dans la situation $^{6}, \ldots$ Pour Kleven (1991), ces connaissances se développent au fur et à mesure de l'expérience d'enseignement dans la classe actuelle et/ou dans des classes précédentes et futures, mais sont aussi le résultat de la formation initiale ou continue de l'enseignant. Les variables émotionnelles renvoient à l'attitude de l'enseignant vis-à-vis de l'enseignement, de la matière à enseigner, ainsi qu'à leurs attitudes à l'égard des élèves.

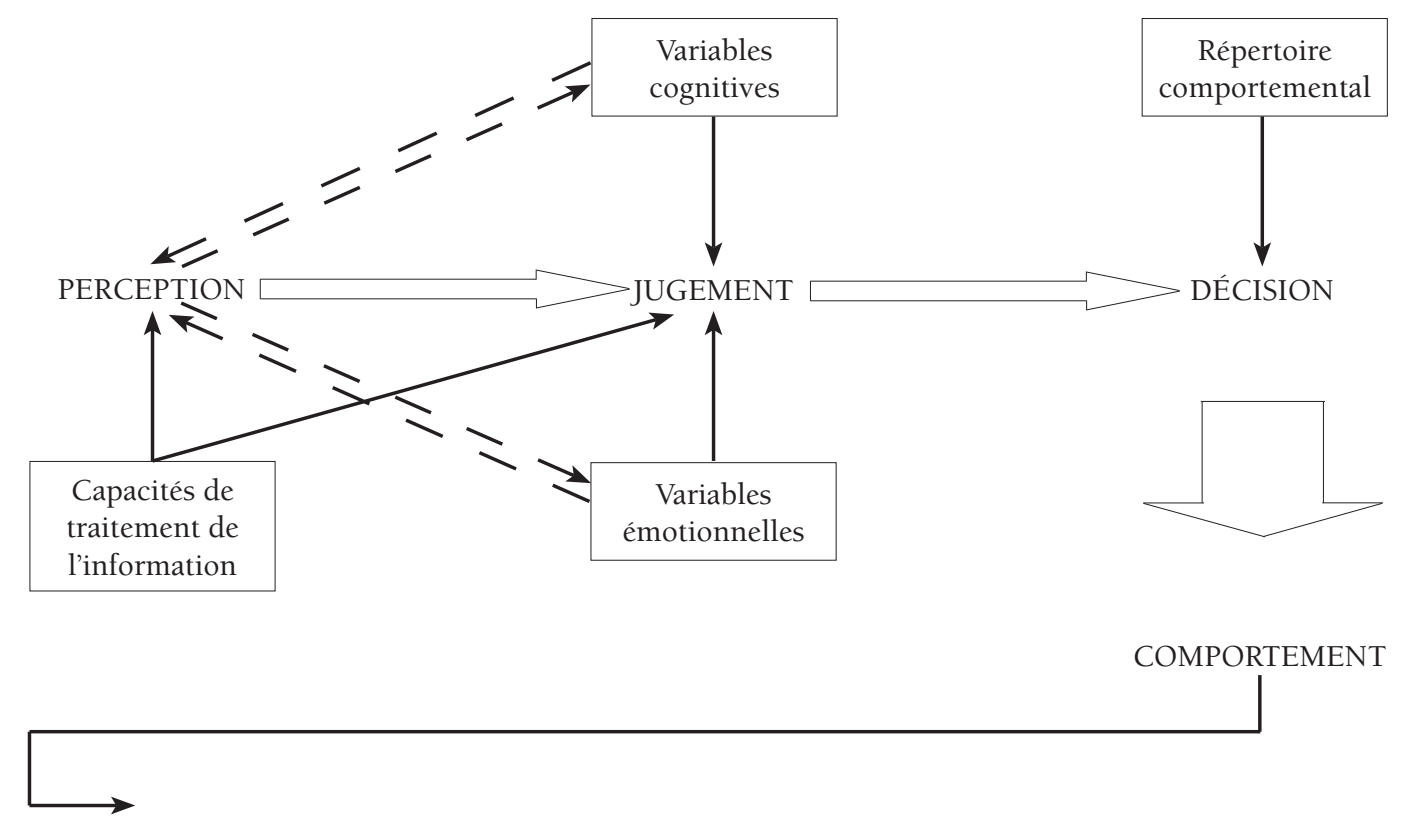

Figure 3: Un modèle général de sens commun d'une situation de décision interactive (Kleven, 1991)

Le jugement amène les enseignants dans une phase de choix d'une action issue du répertoire comportemental. Lors de cette phase, l'enseignant choisit un comportement au sein de son répertoire comportemental qu'il considère comme étant le plus approprié à la situation. Vient enfin la phase d'implémentation de l'action. Le modèle de Kleven (1991) considère donc que l'enseignement interactif consiste en une série de décisions qui se superposent partiellement. Les comportements de l'enseignant sont 
perçus par les élèves qui jugent la situation et choisissent leurs comportements. Les comportements des élèves entraînent une nouvelle situation que l'enseignant perçoit, juge et dans laquelle il décide à nouveau.

Pour Kleven (1991), la phase interactive peut être vue comme une série d'événements ou de situations qui se suivent et se déroulent simultanément. L'enseignant a toujours la liberté de choisir son comportement dans ces situations et peut être considéré comme étant impliqué dans un processus de prises de décisions continuel pendant la phase interactive.

\section{Critiques générales des modèles de la pensée interactive}

Les modèles de Peterson et Clark (1978) ou de Shavelson et Stern (1981) comportent une forte composante managériale. Ils présupposent que la compréhension et la participation des élèves fluctuent au sein d'un espace de tolérance et que l'enseignant perçoit la nécessité d'une décision uniquement quand l'activité des élèves sort des normes d'acceptabilité, celles-ci n'étant précisées par aucun auteur. Selon nous, ce présupposé est caduc à plusieurs niveaux. Premièrement, il n'est pas sûr, conformément à ce que nous verrons par la suite, que le comportement des élèves, même lorsqu'on prend en compte leur compréhension et leur participation, soit le seul antécédent des prises de décisions interactives ${ }^{7}$. Ainsi, il peut aussi y avoir référence au temps, au matériel, etc. Deuxièmement, selon ces modèles, la prise de décision de l'enseignant est fortement contextualisée: un enseignant confronté à plusieurs facteurs prend une décision circonstancielle; une autre fois confronté aux mêmes facteurs, il n'est pas sûr qu'ils reprennent la même décision. (Borko \& Shavelson, 1990; Clark \& Peterson, 1986). Troisièmement, la recherche a montré que la considération d'alternatives par les enseignants est peu fréquente (Marland, 1977; Shroyer, 1981). Dès lors, poser que les décisions interactives produisent la modification du plan par application d'une alternative routinisée est problématique. Quatrièmement, rien ne prouve que la prise de décision des enseignants fonctionne selon un mécanisme de tout ou rien: pas de processus décisionnel lorsque la parti- cipation des élèves est acceptable; prise de décision lorsqu'un seuil est dépassé. Certains auteurs (Borko \& Shavelson, 1990) imaginent un processus s'apparentant à une relation asymptotique du type $U$ renversé: il y aurait une limite inférieure et une limite supérieure définissant une zone optimale au sein de laquelle les enseignants opèrent de façon planifiée et routinisée; en deçà et au-delà de cette zone, le fonctionnement interactif planifié n'est plus possible et des décisions doivent être prises. Cinquièmement, il semble bien que les enseignants prennent certaines décisions pour lesquelles ils n'ont pas de justification (Wanlin, 2009b, 2009c). Sixièmement, on peut reprocher à ces modèles le fait qu'ils représentent la réflexion interactive des enseignants comme procédant de façon algorithmique c'est-à-dire essentiellement rationnelle. Or, les processus cognitifs des enseignants ne sont probablement pas de nature aussi linéaire et mécanique. Brehmer (2005) propose une alternative à cette conception algorithmique; selon lui, les pensées enseignantes seraient composées de modules analytiques rationnels et de modules automatiques routinisés. Par ailleurs, il est probable que des décisions interactives soient prises sur la base de processus principalement émotionnels, une éventualité totalement négligée par les modèles évoqués ci-dessus. En définitive, comme d'autres avant nous, nous doutons que ces modèles proposent une représentation fidèle des processus de prise de décision des enseignants en cours d'instruction (Borko $\&$ Shavelson, 1990; Clark \& Peterson, 1986).

Clark et Peterson (1986) proposent plusieurs recommandations pour affiner les modélisations de la pensée interactive des enseignants. Pour eux, les modèles devraient être révisés dans quatre directions de manière à prendre en compte les constats de la recherche.

1. Les modèles futurs devraient refléter la définition de décision interactive selon laquelle celle-ci consiste en un choix délibéré d'implanter une action spécifique. Autrement dit, il s'agirait de rompre avec l'idée que les décisions interactives sont des choix d'actions parmi une panoplie d'alternatives.

2. Ils devraient aussi refléter le constat que certaines décisions interactives peuvent être précédées par des facteurs relatifs aux élèves et d'autres déterminées par des facteurs relatifs à l'environne- 
ment, aux états de pensée des enseignants ou l'àpropos d'une stratégie d'enseignement particulière.

3. Ils devraient s'intéresser aux processus par lesquels une condition antécédente donnée aboutit à une décision interactive. Dans ce cadre, Clark et Peterson (1986) proposent d'envisager des relations curvilinéaires avec un seuil de réactivité.

4. Ils devraient croiser les catégories d'analyse du contenu des pensées avec des catégories plus spécifiques de processus cognitif telles que celles proposées par Marland (1977) : perception, interprétation, anticipation et réflexion. Bref, Clark et Peterson (1986) encouragent à adopter une vision combinant les modèles décisionnels classiques et celui de Kleven (1991). Cependant, ce dernier modèle n'est pas, non plus, exempt de critiques les définitions de ses composantes sont floues; les opérations principales perception $\rightarrow$ jugement $\rightarrow$ décision, qui constituent son épine dorsale ne sont pas explicitées, il laisse peu de place à l'innovation comportementale des enseignants; il n'est pas immunisé contre la critique de la prégnance managérial, etc.

Tout en y ayant contribué, Clark et Peterson (1986) pensent que la modélisation de la pensée interactive a conduit la recherche dans une fausse direction parce qu'elle postule, d'une part, que le comportement des élèves est l'unique condition antécédente des décisions interactives de l'enseignant et, d'autre part, que celui-ci considère plusieurs alternatives, stratégies ou cours d'action possibles quand il décide de changer ou non son plan d'activité. Même si nous rejoignons leur avis sur la limite que représente la seule référence aux comportements des élèves, nous ne partageons pas cette position radicale. Les modèles de Shavelson et Stern (1981) et de Kleven (1991) présentent des avantages sur lesquels nous nous appuierons pour, en quelque sorte, réhabiliter, au moins partiellement, la voie des modèles décisionnels; ceux-ci ont le mérite de prendre en compte le processus de perception, l'incorporation de l'idée de routine et l'implémentation pendant l'interaction d'un plan mental de l'activité défini lors de la planification. Ces idées permettent de structurer la suite de ce texte pour proposer un modèle intégré de la réflexion en action en passant par le contenu de la pensée interactive et les facteurs qui l'influencent.

\section{Les contenus de la pensée interactive et les facteurs qui les influencent}

La plupart des préoccupations de la pensée interactive ont été définies lors des études portant sur la planification de l'enseignement. De ce fait on peut relever un parallélisme étroit entre les éléments que nous discutons ici et les préoccupations de la pensée planificatrice dont nous avons traité dans une autre publication (Wanlin, 2009c). Notons d'emblée que pour la pensée interactive les découpages entre les préoccupations et les facteurs d'influence dans la littérature sont moins nets qu'ils ne le sont pour la pensée en situation de planification d'activités d'enseignement.

\section{À quoi pensent les enseignants lors de la phase interactive?}

Les études sur le contenu de la pensée interactive ont abouti à des constats convergents. Cette convergence est remarquable car elle émerge d'études variant sur plusieurs points: utilisation du rappel stimulé selon des variantes différentes, investigation auprès de sujets ayant une ancienneté différente, observation à des degrés scolaires différents et sur des branches ou sujets de matière différents; les méthodes de codage et de traitements sont cependant semblables. On peut ranger, selon nous, les constats de la littérature en trois ensembles de préoccupations: le pédagogique, les transitions ainsi que la gestion et l'organisation.

\section{Les préoccupations pédagogiques}

Ces préoccupations renvoient au cour de l'activité d'enseignement apprentissage, c'est-à-dire aux objectifs, aux stratégies, au contenu, au matériel, aux tâches et à l'évaluation. Or, à ce propos, la recherche montre que les enseignants n'allouent qu'une faible portion de pensée interactive au traitement des objectifs d'enseignement et du contenu d'apprentissage, mais en consacrent une grande part au traitement des stratégies ou procédures d'enseignement et des tâches ou activités d'apprentissage (Alexandersson, 1994; Calderhead, 1996; Colker, 1982; Conners, 1978; Lowyck, 1980; Marland, 1977; Marx \& Peterson, 1981; McNair, 1978; Peterson \& Clark, 
1978; Peterson, Marx, \& Clark, 1978; Semmel, 1977; Wanlin, 2009b, 2009c; Warner, 1987), sans oublier les stratégies de questionnement et la sélection des répondants (Marland, 1977; Warner, 1987).

Selon McLeod (1981), les objectifs, quoique peu mentionnés lors des rappels stimulés, jouent cependant un rôle primordial lors de l'interaction puisqu'ils servent de fil rouge auquel les enseignants se réfèrent lors de l'instruction. Les préoccupations pédagogiques consistent notamment en des sélections d'exemples appropriés, des modifications du plan de leçon prévu, en des choix des contenus des feedbacks à adresser aux élèves, etc. (J. Putnam \& Duffy, 1984). Or, on peut considérer que pareilles décisions ne peuvent être prises qu'en fonction d'objectifs à atteindre et d'un contenu à faire assimiler par les élèves, ce qui va dans le sens de la réflexion de McLeod (1981).

\section{Les préoccupations transitionnelles}

Ce type de préoccupation renvoie aux réflexions des enseignants portant sur le pilotage du temps instructionnel et de la fluidité du processus d'enseignement (Arlin, 1979; Wanlin, 2009b, 2009c). Ainsi, selon Arlin (1979) reprenant une idée chère à Kounin (1970), les enseignants cherchent à offrir un rythme et un flux d'activités soutenus à leur enseignement ainsi qu'à minimiser les moments creux lors des transitions entre les activités ou les leçons. Les transitions doivent donc être bien réfléchies, car elles peuvent être à l'origine de perturbations du flux d'activités engendrant l'augmentation de la probabilité d'apparition de comportements déviants de la part des élèves (Arlin, 1979). Le « cadençage » et la "séquenciation » seraient, de ce fait, deux des plus grandes préoccupations des enseignants en cours d'enseignement puisqu'ils déterminent la profondeur et l'étendue de l'apprentissage réalisé en classe (Arlin, 1979; Prosner, $1987^{\circ}$ ). Ces deux préoccupations majeures des enseignants renvoient à leur compétence en matière de gestion du temps et sont, de ce fait, connectée à la prise en compte d'un steering group (Arlin, 1979; Barr \& Dreeben, 1977), notion sur laquelle nous reviendrons plus loin.
Les préoccupations managériales et organisationnelles

Ces préoccupations renvoient aux réflexions des enseignants concernant l'organisation sociale des situations d'enseignement apprentissage; elles incluent la gestion de la discipline et de la classe, de groupes d'élèves ou d'élèves individuels au sein de la classe (Wanlin, 2009b, 2009c). La recherche montre que les décisions managériales et organisationnelles semblent être plus fréquentes en début d'année quand les enseignants n'ont pas encore établi de routines connues de leurs élèves pour structurer et implémenter avec succès les activités (J. Putnam \& Duffy, 1984). Cependant, elles resteraient présentes dans le chef des enseignants tout au long de l'année scolaire, mais dans des proportions moindres (Barr, 1974, 1975).

\section{Quels éléments influencent la pensée interactive?}

Pour moduler leurs réflexions concernant les préoccupations de tout ordre, les enseignants se réfèrent à trois ensembles de facteurs d'influence, dont certains renvoient à l'enseignant, d'autres aux élèves et d'autres encore qui, ne relevant pas de cette dyade, seront qualifiés de contextuels. Notons d'emblée que la recherche montre que les facteurs influençant les préoccupations des enseignants varient de façon idiosyncrasique (Peterson et al., 1978) et que les enseignants se réfèrent à une multiplicité de facteurs pour moduler leurs réflexions interactives (Mahnaz, 1994; Wanlin, 2009b, 2009c).

\section{Facteurs contextuels}

Cette catégorie comprend des facteurs tels que l'environnement matériel, le niveau de l'école, les évaluations externes, les interruptions par des autres adultes (Hofer \& Dobrick, 1981; Lam \& Kember, 2006; Lundgren, 1987; Wodlinger, 1980). On peut également y intégrer l'implémentation de nouveau matériel ou de tâches nouvelles (Hashweh, 2003; Herbst, 2003; Nyroos, 2008) ainsi que des facteurs 


\section{LA PENSÉE DES ENSEIGNANTS PENDANT L'INTERACTION EN CLASSE}

Philippe Wanlin \& Marcel Crahay

sociaux tel que le regard des collègues et de la société (Kansanen et al., 2000). Pour Lampert (1986), l'enseignant est certes influencé par le milieu, mais il le structure lors de sa réflexion qu'il traduit en actes durant l'interaction. Bien que tous ces éléments sont supposés influencer d'une manière ou d'une autre la réflexion interactive, l'essentiel de la littérature mentionne la prise en compte des ressources curriculaires, du temps et des décisions planificatrices.

Comme le rappellent Gamoran et Dreeben (1986), les instances responsables de l'éducation (généralement des ministères) influencent les contenus instructionnels par l'intermédiaire des prescriptions curriculaires officielles; ce faisant, ils influent sur l'allocation du temps scolaire aux différentes disciplines, sur les objectifs poursuivis par les enseignants, sur le matériel disponible et sur la répartition des élèves dans les écoles notamment et, finalement, sur l'apprentissage des élèves. D'une manière générale, il apparaît que le matériel et l'équipement pédagogiques ainsi que les programmes et les manuels scolaires influencent les réflexions interactives des enseignants (Lundgren, 1987, 1972; Marland, 1977; Peterson et al., 1978; Smith \& Geoffrey, 1968; Wodlinger, 1980). Cependant, ces ressources curriculaires n'affectent pas unilatéralement la pratique des enseignants: plus exactement, ceux-ci utilisent ces ressources en les ajustant à leur perception de la situation dans laquelle ils enseignent; ils sélectionnent parmi ces ressources celles qu'ils jugent pertinentes eu égard à leur interprétation des objectifs à privilégier et aux besoins des élèves. Il y a donc un processus de filtrage et d'interprétation par les enseignants des ressources curriculaires. Ainsi, Remillard (2005) montre que l'utilisation du matériel curriculaire et du programme varie selon les enseignants. De surcroît, on observe, chez chaque enseignant, une importante flexibilité, que ce soit lors de la planification ou de l'interaction. Cette flexibilité, notée par de nombreux chercheurs (cf. ci-dessous) a nourri l'idée que le fonctionnement des enseignants (en particulier dans la phase active) est de l'ordre de l'improvisation, semblable en cela aux acteurs, musiciens de jazz, etc. (cf. Erickson, 1982; Tochon, 1993; Yinger, 1987).

La prise en compte du temps et, par extension du rythme, lors des réflexions en action renvoie aux transitions internes, au temps disponible pour terminer la leçon, aux contraintes temporelles liées à l'agenda ou l'horaire (Hofer \& Dobrick, 1981; Lundgren, 1987; Marland, 1977; Tochon, 1990 ; Wodlinger, 1980). Les attitudes envers le temps varient en fonction du profil individuel des enseignants sans nécessairement être liées à leur expérience ou à leur compétence professionnelle (Tochon, 1990). L'impact des instances officielles concernant l'horaire semble, toutefois, puissant. À ce propos, Nyroos (2008) parle d'un effet de rémanence. Elle constate, en effet, que des équipes éducatives invitées à fixer en toute liberté les plages horaires et les durées des cours et de déterminer l'importance en terme de quantité des différentes matières, reproduisent finalement les prescriptions officielles.

Les décisions planificatrices sont probablement la base de toutes les réflexions en action des enseignants; autrement dit, la pensée interactive est grandement influencée par la planification et, en quelque sorte, dépendante de celle-ci (Borko \& Shavelson, 1990; Calderhead, 1996; Clark \& Yinger, 1978; Mahnaz, 1994; Parker \& Gehrke, 1986, 1984; Peterson et al., 1978; J. Putnam \& Duffy, 1984; Tochon, 1993b, 1993a; Warner, 1987). Bien que l'étude de Peterson et Clark (1978) indique une application majoritairement inchangée du plan suggérant que les enseignants restent fidèles à leur planification, plusieurs recherches montrent que les enseignants sont flexibles dans l'implémentation du plan établi lors de la phase préactive (p. ex. : Tochon, 1990, 1993a, 1993b; Tochon \& Dionne, 1994). Le fonctionnement improvisationnel, qui caractérise la pensée interactive des enseignants, renvoie au fonctionnement adaptatif et analogique de l'enseignant ainsi qu'à la nature des processus mentaux lui permettant de s'adapter au contexte de la classe dont il est classique de souligner le caractère mouvant et imprévisible. Pour Tochon (1993a, 1993b), l'improvisation dans l'enseignement est probablement la meilleure, sinon la seule manière pour l'enseignant de prendre en compte les réactions des élèves, dont certaines peuvent être surprenantes, et les événements imprévus de la classe tout en gardant le cap défini par le plan de leçon. La planification écrite serait donc rarement totalement respectée par les enseignants chevronnés, sinon dans les grandes lignes. Le fonctionnement improvisationnel serait donc salutaire car il permet de tenir compte du niveau et des réactions des élèves et semble, dans 
l'esprit des enseignants, accroître la motivation des élèves en maintenant l'atmosphère de travail (Tochon, 1993a, 1993b).

\section{Facteurs propres à l'enseignant}

La recherche montre que les états émotionnels de l'enseignant peuvent influer sur leurs réflexions interactives (Clark \& Yinger, 1978; Wodlinger, 1980). Ainsi, les sentiments personnels des enseignants auraient notamment un impact sur leurs jugements du niveau de compréhension des élèves et sur les stratégies qu'ils choisissent d'utiliser pour l'optimiser (Vagle, 2009). Bien que la recherche indique souvent que les enseignants, même en début de carrière, se réfèrent peu ou pas aux aspects disciplinaires ou didactiques de leur formation initiale, leur préférant plutôt des conceptions de sens commun (Fischler, 1994), certains constatent des effets de la formation des enseignants sur les réflexions interactives (Lam \& Kember, 2006). L'expérience professionnelle combinant des éléments de la vie personnelle des enseignants, dont leurs relations interpersonnelles dans leur vie privée (mariage, enfants,...), leurs caractéristiques personnelles (intérêts, expérience, santé, culture,...) et leur statut socio-économique (visibilité sociale, finances,...), a également un impact sur leur enseignement, sur leur réflexion pédagogique ainsi que sur leurs relations avec les collègues et la direction (Jiménez \& Gersten, 1999; Kansanen et al., 2000; Marland, 1986; Pajak \& Blase, 1989). De même, l'expérience professionnelle engrangée au cours de la pratique d'enseignement sert aussi de référence aux enseignants quand ils pensent en action (Clark \& Yinger, 1978; Davies, 2003; Kansanen et al., 2000; Mahnaz, 1994; Yinger, 1977). Certains pensent même que le caractère idiosyncrasique de la réflexion pédagogique et des conduites d'enseignement serait dû aux expériences professionnelles et personnelles spécifiques à l'enseignant (Kansanen et al., 2000).

Selon certains chercheurs (Creemers \& Westerhof, 1982; Kansanen et al., 2000; Leinhardt, 1983; Lowyck, 2003a; Shavelson \& Stern, 1981), avec l'expérience, les enseignants accumulent des routines, des croyances et des connaissances. Au fur et à mesure que celles-ci se stabilisent, les enseignants les mobilisent lors de l'interaction en classe (Creemers \& Westerhof, 1982; Kansanen et al.,
2000; Leinhardt, 1983; Lowyck, 2003a; Shavelson \& Stern, 1981). Par exemple, dans leur revue de la littérature, Borko et Shavelson (1990) expliquent que les enseignants, surtout les plus chevronnés, se réfèrent à des routines pour réfléchir l'action pédagogique et qu'ils appliquent des routines comportementales pour traduire leurs réflexions en actes.

L'expérience professionnelle et personnelle permet aussi à l'enseignant de constituer un répertoire de savoirs et de croyances auxquels il peut recourir pour agir et réfléchir en action (Alexandersson, 1994; Baumert \& Kunter, 2006; Borko \& R. T. Putnam, 1996; Calderhead, 1996; Davies, 2003; Halkes \& Deijkers, 2003; Hofer \& Dobrick, 1981; Lam \& Kember, 2006; Leuchter, 2009; Mahnaz, 1994; Morine-Dershimer \& Reeve, 1994; Munthe \& Thuen, 2009; J. Putnam \& Duffy, 1984; Weinert, Knopf, \& Storch, 1981; Woolfolk Hoy, Davis, \& Pape, $2006^{9}$ ). À noter que pour traiter des croyances, certains auteurs parlent notamment de principes pédagogiques, de théories implicites ou de critères subjectifs (Clark \& Yinger, 1978; Conners, 1978; Elbaz, 1981; Kansanen et al., 2000; Marland, 1977). Les frontières entre savoirs et croyances sont difficilement saisissables, conduisant bon nombre de spécialistes à traiter ces deux termes de manière quasi synonyme (Borko \& R. T. Putnam, 1996; Calderhead, 1996; Fenstermacher, 1994; Pajares, 1992; Woolfolk Hoy et al., 2006) voire à estimer qu'il serait plus judicieux de considérer les savoirs enseignants comme étant essentiellement des croyances (Kagan, 1992). On retiendra simplement, avec ces chercheurs, que les savoirs feraient davantage référence à des éléments factuels ou empiriques alors que les croyances renverraient plutôt à des convictions ou à des idéologies.

Selon la littérature (Calderhead, 1996; Leuchter, 2009; Shulman, 1987; Woolfolk Hoy et al., 2006), les enseignants développent plusieurs types de savoirs ou croyances. Nous nous référons ici à l'architecture de Borko et R. T. Putnam (1996) qui ont simplifié la typologie initialement proposée par Shulman (1987). Ces deux auteurs proposent une typologie des connaissances et croyances enseignantes organisée en trois ensembles.

1. Les croyances ou connaissances pédagogiques générales: ce sont les savoirs et conceptions de 


\section{LA PENSÉE DES ENSEIGNANTS PENDANT L'INTERACTION EN CLASSE}

Philippe Wanlin \& Marcel Crahay

l'enseignant concernant l'enseignement, l'apprentissage et les apprenants qui transcendent les disciplines scolaires. Elles intègrent les connaissances et croyances des enseignants relatives à la gestion de la classe (comment faire travailler ensemble un groupe d'élèves et maintenir son engagement dans les activités), aux stratégies instructionnelles (comment piloter une leçon, comment créer et structurer des environnements formateurs, quelles stratégies ou routines pédagogiques ou interactionnelles utiliser parmi le répertoire professionnel, etc.) ainsi qu'aux apprenants, leur apprentissage et la manière de leur enseigner (comment les élèves pensent-ils et intègrent-ils la matière, comment l'enseignant peut-il contribuer à cet apprentissage). À noter que la recherche montre que ces connaissances ou croyances relatives aux élèves, qui occupent une grande part du répertoire cognitif des enseignants, sont d'une grande importance dans leur agir efficace (Marland, 1993; Mayer \& Marland, 1997).

2. Les croyances ou connaissances disciplinaires: ce sont les conceptions et savoirs des enseignants dans une matière spécifique. Ils rassemblent les connaissances et croyances concernant les faits, les concepts et la terminologie d'une discipline particulière ainsi que ses idées organisatrices, les connexions entre ces idées, les réflexions et arguments qui la fondent, son développement, etc.

3. Les croyances ou connaissances pédagogiques du contenu: il s'agit de la traduction des connaissances et croyances disciplinaires médiatisée, pour favoriser leur enseignement, par les connaissances et croyances pédagogiques générales. Elles englobent les savoirs et conceptions sur les manières de présenter et de formuler la matière disciplinaire pour la rendre compréhensible à des tiers ainsi qu'une compréhension de ce qui rend complexe ou simple l'apprentissage de certains de ces éléments.

Certaines recherches laissent supposer un lien entre des orientations professionnelles ou des profils de croyances différents selon les enseignants et certains comportements instructionnels (Buchmann, 1987, 1986). Ainsi, Huber et Roth (1990) observent que les enseignants qui ont une tolérance plus élevée face à l'incertitude choisissent davantage des méthodes ouvertes centrées sur les élèves alors que ceux qui préfèrent enseigner dans un climat plus sécurisé préfèrent des méthodes centrées sur l'enseignant. Bien que les connaissances et croyances des enseignants engloberaient à la fois des éléments personnels et des éléments partagés par l'ensemble de la communauté enseignante (Verloop, Van Driel, \& Meijer, 2001), il y aurait un recours idiosyncrasique aux connaissances et croyances pour sélectionner les actes d'enseignement, contrôler leurs mises en œuvre et évaluer leurs effets (Halkes \& Deijkers, 2003; Munby, 1983).

En définitive, il semble bien que les expériences personnelles et professionnelles des enseignants, se constituent en savoirs ou croyances, partiellement partagés avec la communauté des enseignants tout en étant idiosyncrasiques. Celles-ci opèrent en tant que filtres ou, plus précisément, en tant que schèmes permettant aux enseignants d'analyser les situations et de les gérer. Ce mécanisme peut intervenir au moment de la planification de l'enseignement ou dans le courant des phases active et post active (Borko $\&$ Shavelson, 1990; Calderhead, 1996; Woolfolk Hoy et al., 2006). Ainsi, comme indiqué ci-dessus, le curriculum n'affecte pas directement ce qui se passe en classe: son influence est médiatisée par les schèmes cognitifs des enseignants en interaction avec leurs perceptions relatives aux élèves (Remillard, 2005).

\section{Facteurs renvoyant aux élèves}

Les jugements des enseignants portant sur leurs élèves ont une grande influence sur leurs réflexions et comportements interactifs (Borko \& Shavelson, 1990; Clark \& Peterson, 1986 ; Clark \& Yinger, 1978, 1979; Hoge \& Coladarci, 1989; Lissmann, 1989, 2005; Shavelson \& Stern, 1981; Tochon, 1993a). Selon Borko et Shavelson (1978), les enseignants se réfèrent à des facteurs spécifiques relatifs aux élèves pour former leurs jugements et prendre des décisions y correspondant. En définitive, les recherches concernant les facteurs "élèves » pris en compte dans les jugements des enseignants peuvent être regroupées en trois grandes catégories: l'engagement dans les activités d'enseignement, la performance et la compréhension et, enfin, les facteurs complémentaires.

Certaines études montrent que les enseignants se réfèrent à des indices tels que la participation, les comportements non scolaires, l'inattention, les 
états affectifs, les réponses ou le travail incorrects, non satisfaisants ou incomplets de la part des apprenants ainsi que la déviance ou les contacts initiés par les élèves eux-mêmes (Alexandersson, 1994; Arlin, 1979; Bressoux, 2002; Bressoux \& Pansu, 1998; Cadwell \& Jenkins, 1986; Clark \& Yinger, 1978, 1979; Cone, 1978; Doyle, 1979; Fischler, 1994; Fogarty et al., 1982; Gilly, 1992; Housner \& Griffey, 1983; Kansanen et al., 2000; Kounin, 1970a, 1970b; Kounin \& Gump, 1974; Mahnaz, 1994; Marland, 1986, 1977; Natriello \& Dornbusch, 1983; Pullis \& Cadwell, 1982; Tournaki, 2003; Warner, 1987; Wodlinger, 1980). Pour certains chercheurs, les enseignants rechercheraient quasi exclusivement la participation des élèves (Abrahamson, 1974; Doyle, 1979; Westbury, 1973, 1979).

D'autres études montrent, quant à elles, que les résultats d'apprentissage, le niveau de compétences ou de compréhension des élèves influent de façon importante sur la réflexion en action. Ainsi, par exemple, les préoccupations pédagogiques seraient directement influencées par la perception de la part de l'enseignant de la progression des performances scolaires des élèves (Bressoux, 2002; Bressoux \& Pansu, 1998; Cadwell \& Jenkins, 1986; Clark \& Yinger, 1979; Gilly, 1992; J. Putnam \& Duffy, 1984; Tournaki, 2003). La prise en compte des performances scolaires peut se faire à partir d'élèves individuels (Colker, 1982; Conners, 1978; MacKay \& Marland, 1978; Mahnaz, 1994; Marland, 1986, 1977; Marx \& Peterson, 1981; McNair, 1978; Morine-Dershimer, 1978a; Semmel, 1977; Warner, 1987) ou de petits sous ensembles d'élèves de la classe (Arlin, 1979, 1984a, 1973, 1982, 1984b; Arlin \& Webster, 1983; Arlin \& Westbury, 1976; Barr, 1974, 1975; Barr \& Dreeben, 1977; Bromme, 2005, 1989, 1987; Burns, 1987; Dahllö, 1971, 1967; Dahllöf \& Lundgren, 1970; Good, Grouws, \& Beckerman, 1978; Lundgren, 1987, 1972, 1973, 1977; Prosner, 1987; Resnick, 1977; Wanlin, 2009b, 2009c; Warner, 1987).

Pour Dahllöf (1971, 1967), Barr et Dreeben (1977) ainsi que Bromme (2005, 1989), la classe est composée d'une multitude d'individualités d'élèves qui varient quant à leur motivation, leurs intérêts, leurs compétences. Cependant, tous les élèves n'ont pas le même poids dans les réflexions pédagogiques des enseignants. Bromme (2005), d'une part, et Hofer
(1981) d'autre part soutiennent que, le plus souvent, les enseignants ont tendance à regrouper les élèves dans des entités cognitives. Ceci est mis en doute par Oldenbürger (1986). Ce chercheur a répliqué la recherche de Hofer (1981). Or, alors que ce dernier observait un pattern de regroupement, Oldenbürger ne retrouve pas cette tendance chez les enseignants. Ses résultats indiqueraient plutôt que ceux-ci prennent en considération les élèves de façon individuelle. Cette observation va à l'encontre de la notion de steering group introduite par Dahllöf $(1971,1967)$ ainsi que par Dahllöf et Lundgren (1970). Cette notion désigne un ensemble d'élèves de la classe auquel l'enseignant se réfère pour piloter l'allure et la cadence du processus d'enseignement apprentissage ainsi que pour calibrer la difficulté des activités (Barr \& Dreeben, 1977; Bromme, 2005, 1989; Lundgren, 1987, 1972, 1973, 1977; Morine-Dershimer, 1983; Morine-Dershimer \& Tenenberg, 1981; Wanlin, 2009b, 2009c). Le critère principal constituant ce groupe serait, d'après Dahllöf et Lundgren (1970), la performance et le degré de compréhension des élèves. L'existence de ce groupe de pilotage basé sur la performance a été confirmée par Dahllöf (1967, 1971) et par Lundgren $(1972,1973)$ en utilisant des regroupements basés sur des scores de tests standardisés. Barr (1974, 1975), Barr et Dreeben (1977) et Wanlin (2009b, 2009c) aboutissent également à la conclusion que les enseignants opèrent des regroupements d'élèves sur la base de leurs jugements ou d'impressions informelles et, dans la foulée, ont recours à un steering group dans la gestion des activités d'enseignement.

Bien que la recherche indique que la compétence et la performance sont, parmi les caractéristiques des élèves, celles qui affectent le plus les pronostics de réussite formulés par les enseignants ainsi que leurs conduites et réflexions interactives (Clark \& Yinger, 1979; Hofer, 1981; Solomon \& Kendal, 1977; Weinert et al., 1981), Hofer $(2003,1986)$ ainsi que Pullis et Cadwell (1982) montrent que, prises seules, ces variables ne leur suffisent pas pour former un jugement sur leurs élèves. Ainsi, les enseignants utilisent des informations complémentaires comme par exemple les traits de caractère, l'environnement familial, leurs liens avec leurs condisciples, le sexe, le langage corporel, les mimiques faciales, etc. (Clark \& Yinger, 1979; Hofer, 1981; Hofer \& Dobrick, 1981; Tournaki, 2003; Vagle, 2009; Weinert et al., 
1981). Marcoux et Crahay (2008) font des constats analogues concernant les décisions de redoublement: les enseignants se réfèrent aux difficultés d'apprentissage de certains élèves, mais prennent également en considération ses caractéristiques psychologiques ainsi que le soutien que peuvent apporter les parents. Conséquence de ce processus, à performance égale, un élève peut se voir proposer la promotion et un autre de recommencer l'année (Crahay, 2007)

Manifestement, les croyances des enseignants influencent leurs jugements (Aronson, 2004; Barton, 2004; Munthe \& Thuen, 2009; Popham, 2004; Rothstein, 2004; Torff, 2006). Ces derniers adoptent un caractère fortement idiosyncrasique, tant dans le processus de jugement lui-même que dans les variables prises en compte; de surcroît, les jugements des enseignants sont affectés par des paramètres tels que la discipline, la tâche, la situation, le niveau scolaire ainsi que le rôle pris par les sujets (Borko \& Shavelson, 1990, 1978; Cadwell \& Jenkins, 1986; Hofer, 2003, 1986, 2005, 1989; Lissmann, 1989, 2005; Pullis \& Cadwell, 1982). De même, les variables prises en compte divergent selon le type de décision à prendre (Borko et al., 1979; Cadwell $\&$ Jenkins, 1986; Clark \& Yinger, 1978, 1979; Shavelson, Cadwell, \& Izu, 1977; Wodlinger, 1980). Enfin, les enseignants divergent dans l'exactitude de leurs jugements (Clark \& Yinger, 1978, 1979) qui restent, fort heureusement, sujets à révision (Clark \& Yinger, 1978, 1979; Shavelson et al., 1977)

De ce qui précède, on retiendra que le concept de steering group renvoie à une combinaison de jugements des enseignants concernant prioritairement la compréhension et la participation des élèves faisant intervenir, en complément, des éléments contextuels et propres aux enseignants. Cette heuristique servirait non seulement à piloter l'apprentissage des élèves, mais aussi leur attention lors de l'interaction en classe.

\section{Les dilemmes de la pensée interactive}

La gestion simultanée des multiples événements qui font la vie de la classe débouche sur des problèmes quasi insolubles, problèmes qui confrontent les enseignants à des choix cornéliens. Ceci explique que plusieurs recherches aboutissent à la conclusion que la réflexion et l'action des enseignants est gouvernée par la résolution de dilemmes (Ball, 1993; Barr \& Dreeben, 1977; Cohen, 1990; Denicolo, 1996; Herbst, 2003 ; Jackson, 1968 ; Kansanen et al., 2000; Lampert, 1986, 1985, 2001; Lindqvist \& Nordänger, 2006; Parker \& Gehrke, 1986, 1984; Talanquer, Tomanek, \& Novodvorsky, 2007; Tomanek, 1994; Windschitl, 2002).

Les dilemmes sont des situations perçues par l'enseignant comme étant problématiques, dans lesquelles des croyances, des buts ou des indices contradictoires entrent en compétition (Denicolo, 1996; Lampert, 1986; Talanquer et al., 2007 ; Tomanek, 1994). Ces situations sont souvent associées à des insatisfactions liées aux conséquences d'un choix passé ou à celles anticipées pour une décision prise ou à prendre (Denicolo, 1996). Vu l'incompatibilité des éléments qu'elles mettent en tension, ces situations problématiques créent des conséquences jouant en défaveur de l'un ou l'autre de ces éléments (Denicolo, 1996; Herbst, 2003; Lampert, 1986 ; Wagner, 2003; Wanlin, 2009b, 2009c). L'enseignant se perçoit face à un conflit de devoirs insolubles. Ces nouds décisionnels amènent les enseignants à tenter de trouver le meilleur compromis possible entre plusieurs solutions d'intervention alors qu'aucune de celles-ci ne leur paraît vraiment satisfaisante ${ }^{10}$.

Pour Herbst (2003), la notion de dilemme aurait un rôle crucial dans la compréhension des réflexions et des comportements interactifs des enseignants. Elle constituerait l'outil théorique susceptible de rendre compte de la façon dont les enseignants s'efforcent de prendre en charge des ensembles multiples d'obligations pédagogiques et de les transformer en des situations plus simples, gérables par eux lorsqu'ils veulent garantir l'apprentissage et la participation de leurs élèves. L'occurrence de conflits de devoirs insolubles dans l'enseignement serait telle qu'il conviendrait, selon Herbst (2003) mais, avant lui, selon Lampert (1986, 1985), de considérer l'enseignant comme étant un gestionnaire de dilemmes ou un « casseur »d'intérêts contradictoires. Cette obligation de faire face à des dilemmes moraux authentiques serait consubstantielle de l'enseignement, et, par voie de conséquence, serait constitutive de l'identité professionnelle de l'enseignant, de ses connaissances et croyances (Herbst, 2003; Lampert, 1986, 1985; Tomanek, 1994) ${ }^{11}$. 
De façon quelque peu schématique, on peut résumer l'espace de pensée des enseignants, lorsqu'ils gèrent la classe, par trois dilemmes principaux. Le premier, que l'on situera dans le prolongement des travaux de Jackson $(1968,1977,1986)$ ), met en tension la stimulation des apprentissages et le maintien de la collaboration de la majorité sinon de tous les élèves à la vie de la classe. Comme le signale Doyle (1986), il ne s'agit pas d'obtenir de tous un engagement actif dans toutes les tâches, mais il convient que tous acceptent de laisser les activités fonctionner en respectant certaines règles de participation active ou passive. Mais l'enseignant ne peut se contenter de proposer des tâches qui obtiennent l'adhésion du groupe des élèves; celles-ci doivent s'inscrire d'une manière ou d'une autre dans la ligne de programmes en vigueur qui expriment des attentes institutionnelles, eu égard aux compétences à développer chez les élèves. Le second a été baptisé par les Anglo-Saxons le coverage-mastery dilemma; il met les enseignants aux prises avec le rythme de progression dans les apprentissages des différents élèves. Il est en étroite relation avec le troisième dilemme qui, lui, renvoie au pilotage de la cadence de l'enseignement: dès lors qu'il s'agit de gérer les différences individuelles, les enseignants peuvent décider de se concentrer sur les élèves en difficulté en demandant aux meilleurs soit de patienter, soit d'intervenir à titre de tuteurs dans le cadre de travaux en groupe ou, au contraire, ils peuvent décider d'avancer avec ceux qui « ont compris » au détriment des moins rapides. Le choix de se concentrer sur les faibles renvoie à ce que les Anglo-Saxons nomment l'effet Robin-desBois qui, en prenant aux riches pour donner aux pauvres, désavantage les élèves les plus talentueux. Le choix d'avancer avec les «forts » débouche sur l'effet Mathieu qui, lui, défavorise les faibles et/ou les plus lents au profit des plus forts et/ou plus rapides.

\section{Garantir la participation d'un maximum d'élève ou viser l'avancée maximale dans le curriculum (dilemmes du pilotage managérial)}

De nombreuses recherches font état de la présence de cette tension dans le chef des enseignants (Barr \& Dreeben, 1977; Conners, 1978; Doyle, 1986; Herbst, 2003; Jackson, 1968; Peterson \& Clark, 1978; Tomanek, 1994; Yinger, 1980). Pour avancer dans le programme, les enseignants peuvent se contenter de la contribution de quelques élèves, mais ils sont également conscients que leur mission est idéalement d'emmener tous les élèves sur les chemins du savoir. Il semble bien que bon nombre d'enseignants - sinon la plupart - vivent ces deux exigences comme relativement incompatibles. Dans certains cas, eu égard à la composition de la classe, ils perçoivent cette incompatibilité comme radicale.

La littérature fait état d'effets non désirés en relation avec ce dilemme: se sentant contraint de "respecter le programme", les enseignants privilégient les élèves les plus participatifs et les plus compétents au détriment des élèves plus effacés et perturbateurs. Ainsi, Cone (1978) a effectué une étude de type policy capturing; diverses descriptions d'élèves fictifs ont été présentées à 50 enseignants à qui il est demandé de choisir la conduite qu'ils adopteraient face à ces élèves. Ce chercheur constate que:

- Les enseignants sélectionnent davantage de décisions de type managérial pour les élèves auxquels sont associés des comportements déviants et sélectionnent des stratégies managériales sévères dans les cas de violence;

- Ces modes de réactions sont particulièrement activées lorsque le comportement déviant a un caractère récidiviste.

Marland (1977) et Wodlinger (1980) rapportent également des stratégies managériales sévères en relation avec des comportements déviants récidivistes. De façon complémentaire, Cooper, Burger et Seymour (1979) montrent que les interactions avec les élèves forts sont perçues par les enseignants comme étant plus contrôlables que celles avec les élèves faibles. Leurs résultats indiquent également que les enseignants estiment que les interactions avec les forts leur donnent plus de chances, que celles avec les élèves faibles, d'atteindre les objectifs qu'ils se sont fixés en planifiant la leçon. D'autres recherches indiquent que des enseignants font le choix inverse: ils privilégient la participation au détriment du respect du programme, quitte à ne pas voir toute la matière. C'est notamment le sens qu'il faut donner au principe de compensation mis en évidence par Marland (1977) et par Conners (1978) : ce principe, inféré à partir des 
propos de certains enseignants, renvoie au souci qu'ont ceux-ci de favoriser les enfants timides, les introvertis, les moins doués et les défavorisés sur le plan culturel. Dans le prolongement de ces deux études complémentaires (Marland et Conners ont collaboré), Pullis et Cadwell (1982) montrent que les enseignants ont tendance à freiner les élèves qui montrent beaucoup, voire trop, d'entrain lors des activités académiques, ceci afin de préserver un espace d'expression à l'égard des autres. En définitive, ces différentes recherches indiquent clairement que les enseignants ont, en cours d'interaction, des pensées différenciées en fonction des caractéristiques d'élèves. Ils se montrent conscients qu'il leur faut faire des choix pour instaurer des équilibres entre les différents élèves ce qui les amène à freiner certains et, inversement, à « aller chercher » d'autres. Inévitablement, il leur faut décider qui favoriser au détriment de qui.

\section{Achever le programme ou assurer la maîtrise des contenus par un maximum d'élèves (coverage-mastery dilemma ou dilemme lié au pilotage curriculaire)}

Le dilemme du pilotage curriculaire met en tension la gestion par l'enseignant du contenu avec la maîtrise par les élèves des compétences et connaissances enseignées. D'un côté, l'enseignant est conscient des objectifs à atteindre, des prescriptions officielles, du contenu détaillé dans les manuels scolaires et de l'étendue des activités présentées dans le matériel didactique et, de l'autre, il y a son jugement de la compréhension et de la maîtrise effective qu'ont les élèves de ce qui leur a été enseigné. La prise en compte des références curriculaires et des élèves hétérogènes dans leurs aspects cognitifs (niveau de compétence) et non cognitifs (participation, attention, intérêts, etc.) implique que l'enseignant s'investisse dans une réflexion visant à assurer de la cohérence et un certain rythme à son enseignement (Barr $\&$ Dreeben, 1977). Bon nombre d'études mentionnent la difficulté qu'éprouvent les enseignants à choisir entre la maîtrise profonde des objets enseignés par tous les élèves et la couverture des contenus des manuels ou programmes scolaires (Herbst, 2003 ; Lampert, 1986; Talanquer et al., 2007; Tomanek, 1994; Wanlin, 2009b, 2009c).

\section{Avancer avec les forts ou respecter le rythme des faibles (pilotage de la cadence d'enseignement)}

Le pilotage de la cadence de l'enseignement confronte les enseignants à la difficile question de l'adaptation des activités (choisies en accord avec les prescriptions du curriculum officiel) et du rythme de progression au niveau de compétence des élèves. Or, comme déjà indiqué ci-dessus, les élèves diffèrent quant à leur capacité d'assimiler les objets enseignés. Les enseignants sont donc contraints de décider quels élèves prendre en compte pour piloter l'instruction, choisir le niveau de difficulté des activités ainsi que la progression de l'enseignement. Ces décisions font intervenir les jugements des enseignants quant aux capacités de leurs différents élèves (cf. ci-dessus). Ces jugements sont le plus souvent informels, enracinés dans ce qui se passe en situation d'enseignement apprentissage, mais prennent aussi en considération des éléments formels issus des procédures évaluatives. De nombreuses recherches indiquent la présence de ce dilemme dans le chef des enseignants (Barr \& Dreeben, 1977; Herbst, 2003; Talanquer et al., 2007; Woolfolk Hoy et al., 2006). En définitive, ce dilemme amène les enseignants à devoir décider qui des forts ou des faibles serviront de référence pour le pilotage de l'enseignement.

Que l'enseignant se réfère aux forts, aux moyens ou aux faibles, qu'il recoure ou non à un steering group (cf. la discussion précédente), la focalisation sur l'une ou l'autre catégories d'élèves entraîne des effets non désirés (Barr, 1975; Gamoran, 1986; Hofer \& Dobrick, 1981; Rowan \& Miracle, 1983). Ainsi, des recherches confirment que lorsque l'enseignement est piloté en fonction des faibles, les forts sont désavantagés, ont moins d'occasion d'apprendre, sont freinés dans le développement de leurs compétences, doivent sur-apprendre ou sont confrontés à des tâches occupationnelles en attendant la mise à niveau des faibles (Arlin, 1979, 1984a, 1973, 1982, 1984b; Arlin \& Webster, 1983; Arlin \& Westbury, 1976; Barr, 1974, 1975; Barr \& Dreeben, 1977; Burns, 1987; Westbury, 1973). À l'inverse, les faibles sont désavantagés par un pilotage basé sur les forts, comme le montre toute une série de recherches (Aronson, 2004; Barr, 1974; Barton, 2004; Borko \& Cadwell, 1982; Bromme, 2005, 1989; Freeman \& Porter, 1989; Gamoran, 1986; Hofer \& Dobrick, 1981 ; Lujan, 1980; Morine-Dershimer, 1983; 
Morine-Dershimer \& Tenenberg, 1981; Popham, 2004; Pullis \& Cadwell, 1982; Rothstein, 2004; Rowan \& Miracle, 1983; Torff, 2006). On retiendra grosso modo de ces études que les éléments plus "prestigieux " sont réservés aux élèves perçus positivement alors que les éléments moins " prestigieux » sont réservés aux élèves perçus moins positivement. Ces recherches indiquent aussi fréquemment que la qualité des relations pédagogiques diffère selon le jugement de l'enseignant quant à la valeur scolaire de l'élève: par exemple, les relations chaleureuses seraient réservées aux faibles et les stimulations scolaires aux forts. Plus généralement, ces recherches montrent que l'enseignant est face à un dilemme majeur: qui favoriser qui au détriment de qui? Il s'agit bien d'un dilemme car il n'y a pas de choix satisfaisant: les meilleurs ont le droit au meilleur enseignement possible et c'est leur faire subir un préjudice que de les ralentir; parallèlement, négliger les faibles revient à creuser les écarts et, partant, à amplifier les inégalités entre élèves, celles-ci étant souvent d'origine socio-économiques et socioculturelles. Ce dilemme est éminemment idéologique, mais il est aussi au cour des réflexions des enseignants lorsqu'ils enseignent. De ceci émerge une question de recherche qui nous occupera ci-dessous: comment les enseignants gèrent-ils ce dilemme dans le quotidien de leur activité professionnelle.

\section{Des croyances contradictoires à équilibrer}

Certains chercheurs distinguent d'autres dilemmes que ceux énoncés ci-dessus et qui, eux, font - selon notre examen de la littérature - font consensus. Ainsi, nombre de recherches ont montré qu'à l'intérieur des réflexions des enseignants, des convictions contradictoires peuvent coexister et avoir des importances ou des désirabilités égales (Baumert \& Kunter, 2006; Borko \& R. T. Putnam, 1996; Calderhead, 1996; Conners, 1978; Cornett, 1990; Freeman, 1991; Gastager, 2003; Halkes \& Deijkers, 2003; Haritos, 2004; Lampert, 1986 ; Leuchter, 2009; Marland, 1977; Sardo-Brown, 1990 ; Sinatra \& Kardash, 2004; Tomanek, 1994 ; Wagner, 2003; Windschitl, 2002; Woolfolk Hoy et al., 2006; Yildirim, 2003). Par exemple, tiraillés entre leurs croyances qu'un bon enseignement stimule les apprentissages et la participation de tous les élèves et que les leçons nécessitent un rythme soutenu, les enseignants doivent choisir de favoriser l'une de ces conceptions les amenant parfois à devoir mettre en place des stratégies compensatoires sur lesquelles nous reviendrons plus bas. Outre l'implémentation de procédures correctrices, il semblerait que les enseignants peuvent résoudre ces inconsistances dans leurs connaissances ou croyances en créant des convictions intermédiaires consensuelles ou en modifiant leurs croyances et connaissances pour justifier leurs comportements d'enseignement (Tabachnick \& Zeichner, 2003, 1986).

S'agit-il d'une autre catégorie de dilemmes? Nous ne le pensons pas dès lors que ce conflit de croyances peut se résoudre dans ce que Piaget (1975) aurait appelé une équilibration majorante. Plus précisément, dès lors que le conflit de croyances peut se résoudre par un rééquilibrage de la structure de celles-ci, il n'y a plus dilemme au sens propre du terme, lequel renvoie à l'idée d'un conflit d'ordre éthique qui, confrontant des valeurs jugées équivalentes, ne peut trouver de solution pleinement satisfaisante.

En revanche, il nous semble que nous sommes au cœur d'un vrai dilemme dans le cas où des enseignants étudiés par Jiménez et Gersten (1999) se demandent s'il leur faut respecter la culture du système scolaire voire de l'école ou de faire davantage correspondre son enseignement à la culture des élèves notamment à statut socio-économique moins valorisé.

Quelques recherches font état de tensions vécues par des enseignants qui hésitent entre l'action de continuer l'enseignement d'une matière académique et le respect du temps de récréation ou la mise en place d'une matière moins académique lorsque l'atteinte des objectifs de la matière académique n'est pas assurée (Barr \& Dreeben, 1977; Dahllöf, 1971, 1967; Dahllöf \& Lundgren, 1970; Lundgren, 1987, 1977; Parker \& Gehrke, 1986, 1984; Tomanek, 1994). De notre point de vue, on se trouve dans ce cas dans une variante du dilemme mastery versus content coverage en ce sens que ce conflit cognitif oppose la décentration sur l'élève au respect des prescriptions officielles. 


\section{De quelques stratégies mises en ouvre par les enseignants pour atténuer les effets non souhaités de certains choix didactiques}

Par essence, un dilemme n'a pas de solution pleinement satisfaisante. Le résoudre est donc une entreprise impossible. C'est ce que vivent certains enseignants (Tomanek, 1994). Pour ceux-là, la gestion des dilemmes est source d'incertitudes et de sentiments de malaise professionnel (Lindqvist $\&$ Nordänger, 2006). Afin de réduire cet inconfort ou cette insatisfaction, certains s'efforceraient de mettre en place des stratégies de compensation afin de minimiser les effets non désirés des choix didactiques effectuées à certains moments du processus d'enseignement eu égard aux dilemmes désignés ci-dessus.

Dans une sorte de self study, Lampert (1986, 1985) mentionne trois stratégies qu'elle et d'autres enseignants mettent en place pour atténuer la tension de certains dilemmes. Pour l'essentiel, il s'agit de reconsidérer les objectifs poursuivis, de les ajuster voire d'en substituer d'autres plutôt que de tenter de les réaliser sans prise en considération de l'évolution de la situation d'enseignement-apprentissage. Les trois procédures mises en évidence par Lampert (1985, 1986) sont les suivantes:

1. La stratégie de réorganisation sociale consiste à réorganiser le groupement des élèves afin de favoriser leurs apprentissages tout en tenant compte de leurs besoins personnels et psychologiques.

2. La stratégie de négociation consiste, en fait, à renoncer en quelque sorte à imposer une procédure corrective pour éviter que l'élève se rebiffe et se retire de l'activité d'enseignement voire tente de la saborder ${ }^{12}$.

3. La stratégie de réorganisation structurelle avec négociation consiste à proposer des aménagements structurels dans la manière de présenter la leçon (fournir des tableaux abaques, réarrangement de l'espace de travail, préciser davantage les consignes,...) tout en postposant la procédure évaluative pour éviter de devoir confronter trop abruptement certains élèves, plus fragiles, à leurs erreurs. Cette procédure intègre également la suggestion à l'auto-évaluation et à l'autorégulation.
Contrairement à Lampert qui parle de redéfinition d'objectifs, Wanlin (2009b, 2009c) montre, quant à lui, que des enseignants tentent de corriger les effets engendrés par leur choix de piloter l'instruction en fonction d'un groupe donné d'élève en mettant en place de procédures de compensation adressées aux autres groupes d'élèves. Il identifie plusieurs stratégies compensatoires utilisées par des enseignants primaires pour gérer au moins mal le dilemme relatif au pilotage de la cadence d'enseignement. Certaines de ces stratégies sont mises en œuvre de façon complémentaire.

- Le calibrage comprend la personnalisation des sollicitations et le contrôle de leur degré de complexité en fonction du niveau des élèves. Elle permet d'augmenter les interventions de certains élèves et leurs occasions de recevoir des feedbacks essentiellement positifs. On distingue le calibrage de correction surtout adressé aux élèves faibles à qui l'enseignant demande d'améliorer leurs réponses et, le calibrage de développement adressés aux élèves forts qui sont invités à aller au-delà de leurs interventions.

- L'assistance renvoie à une sorte de protection des élèves faibles d'une confrontation individuelle aux exercices plus complexes par l'assurance d'un suivi de leur travail. Elle peut également être adressée aux élèves moins disciplinés dans l'optique de maintenir leur engagement dans la tâche.

- Le stand-by suppose l'attente des élèves rapides qui réalisent des exercices de dépassement ou d'occupation jusqu'à ce que les plus lents aient atteint le niveau désiré par l'enseignant.

- Le contrôle consiste en la canalisation des comportements des élèves sur lesquels ne porte pas la focalisation de l'enseignant. Il ambitionne de maintenir une ambiance disciplinaire propice à l'apprentissage.

Dans une étude suivante, procédant par questionnaire, Wanlin (2010a) constate qu'il se pourrait que des enseignants utilisent deux autres stratégies de compensation. La première consiste à protéger les élèves faibles d'une confrontation directe aux exercices de dépassement des compétences de base. Dans la seconde, l'enseignant invite les élèves forts qui sont 
aussi les plus rapides à réaliser des exercices de dépassement pendant qu'il se concentre sur les plus lents afin d'atteindre avec eux le niveau de compétence qu'il vise pour tous. Il convient, cependant, de rappeler ici les observations réalisées par Arlin (1982) et par Westbury (1973) dans le cadre d'expériences de mise en place de la pédagogie de maîtrise: les exercices destinés aux élèves rapides pendant que les faibles se concentrent sur des exercices supplémentaires seraient plus souvent des tâches d'occupation que des tâches de dépassement des compétences de base.

Il est encore intéressant de mentionner l'étude Parker et Gehrke $(1986,1984)$ qui indiquent la mise en œuvre de procédures comportementales de la part des enseignants visant à assurer le bon déroulement du plan mental. Celles-ci sont notamment les suivantes: accompagnement minutieux des élèves à la traine, encouragement à effectuer le travail plus rapidement, redirection des efforts des élèves par des indices précis, etc. Ces procédures peuvent - nous semble-t-il - être rapprochées des stratégies compensatoires identifiées par Wanlin (2009b, 2009c, 2010a) pour gérer les dilemmes surgissant lors de la réflexion interactive.

Il existe probablement encore d'autres stratégies de compensation. L'objectif ici n'est pas de présenter un relevé exhaustif de ces stratégies. Il s'agit plutôt d'illustrer une facette que nous pensons importante de l'activité des enseignants. Pour atténuer les effets non désirés des choix qu'ils effectuent à un moment $\mathrm{du}$ processus d'enseignement pour faire face aux dilemmes que nous supposons être consubstantiels à toute situation d'enseignement, les enseignants mobilisent des stratégies que nous qualifions de compensation. Plus généralement, nous formulons l'hypothèse que, confrontés inévitablement à des conflits de devoirs insolubles, la plupart des enseignants s'efforcent de minimiser d'une manière ou d'une autre les effets non désirés des choix effectués à certains moments du processus d'enseignement. À nos yeux, cette hypothèse générale mériterait l'intérêt de quelques chercheurs.

\section{Vers un modèle intégré de la réflexion interactive}

La littérature montre que les activités, définies lors de la planification, sont l'unité de traite- ment de base de l'enseignement en classe (Doyle, 1983; R. T. Putnam, 1987; Yinger, 1979) et que les réflexions interactives leur sont subordonnées (Parker \& Gehrke, 1986, 1984). Partant du principe que, lors de la planification de l'enseignement, les enseignants construisent des images mentales des activités à réaliser en classe et que les réflexions interactives sont les médiateurs mentaux par lesquels ils parviennent à les implémenter, on peut proposer, à titre d'hypothèse, un modèle de la réflexion interactive (voir figure 4), ceci en mettant à profit la revue de la littérature qui précède.

Le point de départ du schéma est, vu ce qui vient d'être rappelé, le plan élaboré lors de la phase préactive; celui-ci est présent dans le champ mental de l'enseignant sous forme d'images mentales ou, plus précisément, d'un script de la leçon à implémenter. Comme le montrent diverses recherches (Borko \& Shavelson, 1990; Clark \& Peterson, 1986; MorineDershimer, 1978a; Wanlin, 2009a), quand ils interagissent avec leurs élèves, les enseignants se réfèrent à ces scripts et tentent de leur rester fidèles. Dès lors, en situation de classe, ceux-ci concentrent leurs ressources cognitives sur l'exécution d'un script. Leur but est de mener l'activité à son terme, c'est-àdire d'atteindre leur(s) objectif(s) tel(s) que défini(s) par le plan de leçon. Pour ce faire, ils mobilisent des routines qui, disponibles dans leur répertoire comportemental, ont fait leurs preuves. Dans l'exécution du script, il leur faut tenir compte du temps disponible et en ajuster l'effectuation en fonction du temps écoulé (Borko \& Shavelson, 1990; Leinhardt \& Greeno, 1986; Parker \& Gehrke, 1986, 1984). Avec Leinhardt et Greeno (1986) ainsi que Borko et Shavelson (1990), Nous pensons que les réflexions interactives consistent pour l'essentiel à décider s'il est indiqué ou non de poursuivre le plan mental lors de l'interaction en classe, de l'abréger et/ou d'accélérer la vitesse de son déroulement. Dans ce contexte, certains passages du script feraient office de points de contrôle ayant pour fonction de permettre de réguler ou non le processus d'enseignement. Pour ce faire, les enseignants se basent sur deux paramètres principaux: les steering groups et leurs croyances.

Trois remarques à ce propos:

- Comme nous l'avons vu plus avant, les steering groups peuvent être considérés comme des sortes de 


\section{LA PENSÉE DES ENSEIGNANTS PENDANT L'INTERACTION EN CLASSE}

Philippe Wanlin \& Marcel Crahay

pacemakers ${ }^{13}$ de la classe. Pour composer ces groupes, l'enseignant prend en compte à la fois des éléments renvoyant à la participation des élèves (implication dans la tâche, attention, comportement), à leurs compréhensions (performance, maîtrise,...) et à d'autres facteurs.

- Il apparaît dans la littérature que l'on peut faire l'hypothèse de la coexistence de plusieurs steering groups dans les réflexions enseignantes: les uns servant à cadencer et séquencer l'enseignement et les autres servant à gérer les comportements en classe. De même, il semblerait que la prise en considération de ces groupes soit mobile: l'enseignant privilégiant tantôt l'un, tantôt l'autre.

- La littérature montre que les croyances et les connaissances des enseignants leur servent de filtres pour analyser notamment les situations interactives. Par ailleurs, les croyances renvoient à des pratiques pédagogiques considérées comme adaptées. Les savoirs et conceptions des enseignants interviendraient donc à deux niveaux: lors de la phase d'observation-perception-jugement, ils servent à analyser et juger l'interaction et, lors de la phase de décisioncomportement, ils fournissent un stock de conduites d'enseignement dans lequel les enseignants peuvent puiser pour traduire leurs décisions en actes.

Pendant le processus instructionnel, l'enseignant opérerait un monitoring du déroulement de l'activité, prélevant des indices de deux types. Simultanément, il évaluerait dans quelle mesure l'activité se réalise conformément à son script et à ses croyances pédagogiques et dans quelle mesure les différents groups d'élèves contribuent à l'avancement de la leçon. C'est de ce prélèvement d'indices que peuvent surgir l'un ou l'autre dilemme ou le constat d'un imprévu. C'est également à partir de ce prélèvement d'indices que l'enseignant peut juger qu'il y a dépassement des seuils de tolérance quant à la participation des élèves. Dans ces trois cas, le modus operandi consistant à implémenter la planification sur la base de routines serait interrompu, l'enseignant s'engageant dans un processus de réflexion l'amenant à analyser quel comportement adopter. Rappelons à ce propos que, d'une manière générale, les enseignants rechignent à changer le plan d'activités qu'ils avaient prévu (Borko \& Shavelson, 1990). On peut avancer deux hypothèses pour expliquer cette aversion au changement de script en cours d'interactions en classe: d'une part, les routines sélectionnées lors de la planification seraient la meilleure alternative aux yeux des enseignants et, d'autre part, les modifications apporteraient trop d'incertitudes pour les élèves ainsi que pour l'enseignant (Borko \& Shavelson, 1990; Parker \& Gehrke, 1986, 1984; Shavelson \& Stern, 1981). Il en résulte donc que les changements ne sont que mineurs ou n'amènent qu'un affinement plutôt que d'être des modifications majeures de la planification.

Dans notre conception, la fonction de la réflexion en action n'est pas seulement de mettre en ouvre le plan, mais aussi de réguler le processus instructionnel quand il ne progresse pas (ou plus) de manière suffisamment fluide en référence aux croyances et savoirs des enseignants, ainsi qu'au groupe d'élèves que l'enseignant a pris pour repère pour piloter la cadence de l'activité. Les situations amenant une rupture du modus operandi aboutissent globalement à quatre types de comportements:

- continuer à implémenter le plan sans y apporter de changement;

- contenir les indices de participation et de compréhension à l'intérieur de fourchettes acceptables par rapport aux seuils de tolérance propre à l'enseignant (Brophy \& McCaslin, 1992);

- adapter le plan mental, généralement de manière légère, en ne créant pas trop d'incertitude (souvent par l'utilisation de routines ayant fait leurs preuves, mais la possibilité d'essais de nouvelle procédure n'est pas exclue);

- arrêter l'enseignement de la séquence d'activités.

On pourrait émettre l'hypothèse que les enseignants recourent, dans la plupart des activités (sinon dans toutes), à la stratégie consistant à contenir les indices pour implémenter les scénarii fixés lors de la planification. Cette hypothèse est, en tout cas, cohérente avec l'observation de Parker et Gehrke (1986), reprise par Borko et Shavelson (1990): les activités qui sont sélectionnées lors de la planification ont la propriété d'être le plus souvent conduites jusqu'à l'atteinte des objectifs qui leurs étaient assignés. 


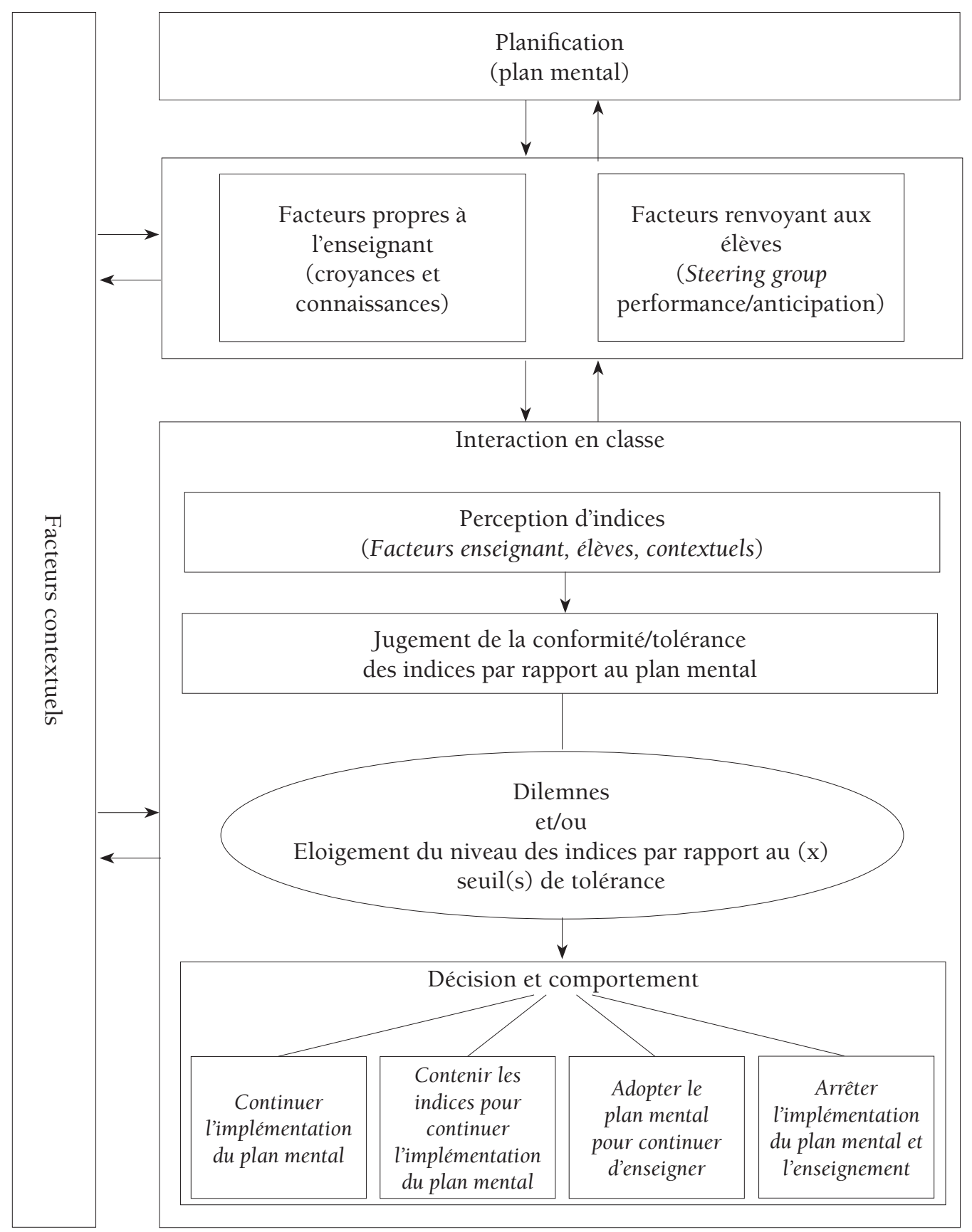

Figure 4: Modèle intégré de la pensée interactive

\section{Discussion}

Le concept de steering group permet de voir l'enseignant comme un coordinateur alerte et dynamique $\mathrm{du}$ processus instructionnel, qui ajuste son contenu en fonction des ressources curriculaires et des objec- tifs qu'il s'est fixés en planifiant la leçon. Pour ce faire, il prélève constamment des indices quant à l'implication des élèves dans l'activité. À ce propos, rappelons que trois théories de relevé d'indices «élèves » ont grosso modo coexisté dans la littérature. La première conception, que nous rapprocherons des travaux 
de Dahllöf et de Lundgren, relie la progression des apprentissages des élèves directement à deux compétences de l'enseignant: l'observation des indices de compréhension des élèves, d'une part, et d'autre part, la sélection des concepts important des contenus enseignés ainsi que l'ajustement des questions au niveau des élèves. Cette conception renvoie donc à l'idée selon laquelle la pensée interactive est rationnelle et que l'enseignant pose des questions et évalue les élèves de manière réfléchie en se référant essentiellement à leurs performances. La deuxième théorie renvoie à l'idée de Jackson (1968) selon laquelle les enseignants chercheraient surtout à maintenir l'engagement des élèves dans les tâches instructionnelles. Dans cette conception, l'élément déterminant quant à la transition d'une activité à l'autre n'est pas le niveau de compréhension des élèves, mais les impressions de l'enseignant concernant l'ennui potentiel de ses élèves face aux contenus en cours d'instruction, leur motivation ou leur participation. La troisième théorie renvoie aux travaux de Kounin (1970a, 1970b), Snow (1972), Peterson et Clark (1978) ou Shavelson et Stern (1981) qui basent les réflexions interactives sur l'observation du comportement des élèves lesquels sont essentiellement jugés à l'aune de leur déviance ou non par rapport à des normes d'implication dans la tâche. Il s'agit, dans ce cas, d'une conception managériale, la prise en considération des élèves étant essentiellement liée à la gestion de la discipline en classe.

Ces trois conceptions paraissent aujourd'hui toutes partiellement correctes, mais incomplètes. Il paraît difficile actuellement de prétendre que, pour piloter la classe, l'enseignant se réfère uniquement à la compréhension, ou exclusivement à la participation ou seulement aux comportements « a-scolaires » des élèves. Selon nous, la réalité pédagogique se situe à l'intersection de ces trois conceptions. L'enseignant s'efforce simultanément de maintenir les élèves au travail et de déterminer le rythme de progression instructionnelle, tout en assurant un climat de classe propice à l'apprentissage. Ceci le contraint à rechercher les façons de regrouper les élèves de manière pertinentes lors des travaux en équipe, de sélectionner des activités d'apprentissages pertinentes, etc. La prise en compte simultanée de ces éléments a notamment été documentée par Alexandersson (1994) ainsi que par Parker et Gehrke (1986, 1984) qui, en tentant d'analyser la différence entre les déci- sions relatives à la gestion et celles concernant le contenu, arrivent à la conclusion que ces deux types de décisions sont intimement liés et pour ainsi dire indissociables. En définitive, l'enseignant s'efforce de faire front à la multidimensionnalité de la vie de classe, cherchant à susciter l'intérêt des enfants ainsi que leurs apprentissages tout en gérant la discipline. Il s'efforce aussi à prendre considération l'hétérogénéité des élèves, ce qui le conduit inévitablement à se confronter à des conflits de devoir insolubles. Ces dilemmes, les enseignants ne semblent pas les affronter en tranchant dans le vif, en faveur d'un groupe ou d'un autre. D'une part, contrairement aux premières études portant sur le steering group, il apparaît que les enseignants ne se concentrent pas sur une seule catégorie d'élève au détriment des autres: ils se dotent de plusieurs steering groups. D'autre part, lorsqu'ils ont le sentiment d'avoir désavantagé un groupe, ils redéfinissent les objectifs ou, le plus souvent, pensons-nous, ils mettent en place des stratégies de compensation.

Toutes ces idées, nous avons tenté de les organiser selon un modèle schématisant le fonctionnement cognitif des enseignants en situation de classe. Ce modèle n'est pas validé. Il convient donc de le considérer comme une heuristique dont la plausibilité provient du fait qu'il a été élaboré au terme d'une revue aussi complète que possible de la littérature existante concernant le teacher thinking. Par rapport à ses prédécesseurs, il présente - nous semble-t-il plusieurs avantages. D'abord, il tient compte du fait que les enseignants se réfèrent à une multitude de facteurs pour réfléchir leurs interventions didactiques. Ces facteurs renvoient non seulement aux élèves, à leur participation aux activités ou à leur compréhension de l'enseignement, mais aussi aux enseignants eux-mêmes, plus précisément à leurs connaissances et croyances. Il tient aussi compte du fait que les enseignants prennent en considération le temps d'enseignement et certains éléments contextuels. En son cœur, il place la notion de dilemme, signifiant par là qu'il y a dans toute situation d'enseignement des choix à faire entre des options incompatibles. C'est peut-être dans cette caractéristique consubstantielle de l'activité d'enseignement qu'il faut chercher la raison de la fréquente insatisfaction professionnelle des enseignants: du point de vue qui est le nôtre, une activité d'enseignement n'est jamais totalement réussie, puisqu'il a fallu soit laisser les 
plus faibles en arrière par rapport aux forts ou qu'il a fallu freiner ceux-ci au profit des moins rapides. Avec Herbst (2003), nous pensons que la notion de dilemme pourrait devenir un construct central de l'étude de l'enseignement. À nos yeux, la façon dont les enseignants y font face mériterait un véritable programme de recherches.

Autre élément clef du modèle présenté ci-dessus, le processus de perception-décision qui s'articule autour des jugements produits par les enseignants quant à la conformité des indices divers prélevés par eux au script élaboré pendant la phase de planification. Ce processus conduit les enseignants à des décisions qui ne se résument pas à choisir entre des alternatives comportementales, mais peut les amener à maintenir inchangé le plan de leçon. Ceci permet de rappeler une autre caractéristique majeure du modèle que nous défendons: il part de l'idée que les réflexions interactives sont largement influencées par les décisions planificatrices effectuées en amont de l'intervention en classe. L'enseignant y est vu comme un acteur qui implémente le plan de leçon qu'il a conçu au préalable. Combiné au modèle de la pensée planificatrice que nous avons proposé ailleurs (Wanlin, 2009c), il donne à voir comment la pensée interactive s'articule avec les réflexions en cours de planification. Cette combinaison va dans le sens de Borko et Shavelson (1990) pour qui la distinction entre pensée planificatrice et interactive proposée initialement (cf. Jackson, 1968) est obsolète et inappropriée. Selon ces auteurs, en effet, les réflexions préactive et interactive ne sont pas des processus distincts; il conviendrait plutôt de les concevoir comme des systèmes interconnectés. C'est d'ailleurs ce que pensent les enseignants qui estiment que planification et interaction sont intimement liées (Lowyck, 2003b).

Bien sûr, nous espérons que des chercheurs pourraient se nourrir de ce modèle hypothétique pour orienter leurs investigations. Cependant, la recherche n'est probablement pas la seule qui pourrait bénéficier de ce modèle avec, en son centre, le concept de dilemme. Nous reprenons ici à notre compte l'avis de Tomanek (1994), de Denicolo (1996) et de Talanquer et al. (2007) qui pensent que l'approche par dilemmes pourrait contribuer à une optimisation de la formation initiale et continue des enseignants. Pour eux, cette notion permettrait de former des enseignants prêts à affronter l'incertitude, l'ambigüité et la multidimensionnalité inhérente à toute activité d'enseignement. 


\section{NOTES}

1. Pour les deux autres pôles voir Wanlin (2009a) et Crahay, Wanlin, Issaieva et Laduron (2010).

2. N'ayant pu trouver de traduction satisfaisante de cette expression, nous conserverons la terminologie anglaise tout au long de ce texte.

3. Ceci n'empêche pas MacKay et Marland (1978) et Lowyck (1980) d'écrire que la prise de décision interactive des enseignants est moins fréquente qu'on ne le pense.

4. Cette étude s'intègre dans un programme plus vaste portant sur les caractéristiques de l'enseignement efficace (see Clark, Snow, \& Shavelson, 1976).

5. Le withitness et l'overlapping sont des concepts dus à Kounin (1970). La première de ces notions renvoie à la vigilance de l'enseignant qui perçoit tout ce qui se produit en tout point de la classe. La deuxième notion fait référence à la capacité de l'enseignant qui parvient à traiter plusieurs éléments en simultané lors de l'interaction en classe.

6. Notons que Kleven (1991) se réfère, à ce sujet, à la typologie de Shulman (1987).

7. Pour Snow (1972), l'antécédent des réflexions enseignantes est le comportement des élèves d'un point de vue disciplinaire. Peterson et Clark (1978), qui se basent largement sur le modèle de Snow, posent que cet antécédent renvoie davantage à la compréhension et à la participation des élèves. Concernant Kleven (1991), bien que son modèle intègre des indices généraux, il s'avère que dans la discussion c'est essentiellement aux comportements des élèves qu'il fait référence. Bref, nos modèles n'échappent pas à la critique dont il est question ici.

8. La littérature définit le cadençage (pacing) comme étant les décisions transitionnelles concernant le rythme instructionnel à adopter, la rapidité de progression à l'intérieur des activités. Il amène donc l'enseignant à devoir choisir s'il doit accélérer ou freiner le rythme instructionnel. La séquenciation (sequencing), quant à elle, est définie dans la littérature comme étant les décisions transitionnelles concernant les enchaînements des activités entre elles que ce soit interne à la leçon (comprise comme une séquence de plusieurs activités) ou entre les leçons (d'une séquence d'activités à une autre).

9. Pour une revue francophone ciblée sur la question, voir Crahay et al. (2010).

10. Il est intéressant de noter que, dans le domaine de l'éthique, la notion de dilemme a mis longtemps à faire son chemin (Lemmon, 1962; Williams, 1965). Sa possibilité a été niée par plusieurs philosophes; parmi eux, Aristote, Thomas d'Aquin, Kant et Mill. À l'opposé, nombreux sont les philosophes contemporains qui admettent la possibilité des dilemmes moraux authentiques. Dans L'existentialisme est un humanisme, Sartre (1964) donne l'exemple d'un élève qui hésite entre rejoindre les Forces françaises en exil, et ainsi venger son père ou rester auprès de sa mère, et l'aider à vivre. Dans Le Choix de Sophie, Styron (1979) donne une place importante aux dilemmes moraux; il traite en particulier l'exemple suivant: dans un camp de concentration, un garde oblige une mère à choisir, entre ses deux enfants, lequel sera tué; il ajoute que si la mère ne choisit pas, les deux seront tués.

11. Notons que les dilemmes conjuguent à la fois des éléments transversaux à l'enseignement et des éléments propres aux différentes disciplines (Chazan, 2000; Chazan \& Ball, 1999; Herbst, 2003; Lampert, 2001), dans la mesure où ils mettent en jeux les connaissances et croyances des enseignants, elles-mêmes composés d'éléments conjuguant pédagogique et disciplinaire (cf. Crahay et al., 2010). 
12. Lampert $(1985,1986)$ raconte que, dans une classe, elle perçut une tension en étant confronté à un élève plutôt passif qui, un jour, se met à participer, mais commet des erreurs de prononciation. L'enseignante, Lampert, en l'occurrence (à noter que Lampert expose également le cas d'autres enseignants dans ses deux textes), craint de blesser en le corrigeant publiquement. Alors, elle se décide à lui fournir des indices différents mais pas correctifs,... Cette décision est également justifiée par le fait que cet élève avait un amour-propre énorme et, lorsqu'il se sentait blessé, il sabotait le cours. Finalement, l'enseignant renonce à faire des remarques et s'efforce d'atteindre des objectifs autres de manière détournée.

13. Il faut entendre cette expression au sens premier des deux termes qui la composent: pace correspondant au rythme et maker à impulseur.

\section{RÉFÉRENCES}

Abrahamson, J. H. (1974). Classroom contraints and teacher coping strategies: a way to conceptualize the teaching task. University of Chicago.

Alexandersson, M. (1994). Focusing teacher consciousness: what do teachers direct their consciousness towrds during their teaching. Dans I. Carlgren, G. Handal, \& S. Vaage (Éd.), Teachers'minds and actions: research on teachers'thinking and practice (p. 139-149). London: Falmer Press.

Arlin, M. (1973). Learning rate and learning rate variance under mastery learning conditions. University of Chicago.

Arlin, M. (1979). Teacher transitions can disrupt time flow in classrooms. American Educational Research Journal, 16 (1), p. 42-56.

Arlin, M. (1982). Teacher responses to student time differences in mastery learning. American Journal of Education, 90 , p. 334-352.

Arlin, M. (1984a). Time, equality, and mastery learning. Review of Educational Research, 54, p. 65-86.

Arlin, M. (1984b). Time variability in mastery learning. American Educational Research Journal, 21, p. 103-120.

Arlin, M., \& Webster, J. (1983). Time costs of mastery learning. Journal of Educational Psychology, 75 (2), p. 187-195.

Arlin, M., \& Westbury, I. (1976). The levelling effect of teacher pacing on science content mastery. Journal of Research in Science Teaching, 13, p. 213-219.

Aronson, J. (2004). The threat of stereotype. Educational Leadership, 62, p. 14-20.

Ball, D. (1993). With the eye on the mathematical horizon: Dilemmas of teaching elementary school mathematics. Elementary School Journal, 93, p. 373-397.

Barr, R. C. (1974). Instructional pace differences and their effect on reading acquisition. Reading Research Quarterly, 9, p. 526-554.

Barr, R. C. (1975). How children are taught to read: Grouping and pacing. School Review, 83, p. 479-498. 


\section{LA PENSÉE DES ENSEIGNANTS PENDANT L'INTERACTION EN CLASSE}

Philippe Wanlin \& Marcel Crahay

Barr, R. C., \& Dreeben, R. (1977). Instruction in classroom. Review of Educational Research, 5, p. 89-162.

Barton, P. (2004). Why does the gap persist? Educational Leadership, 62, p. 8-13.

Baumert, J., \& Kunter, M. (2006). Stichwort: Professionelle Kompetenz von Lehrkräften. Zeitschrift für Erziehungswissenschaft, 4, p. 469-520.

Borko, H., \& Cadwell, J. (1982). Individual differences in teachers‘ decision strategies: an investigtion of classroom organization and management decisions. Journal of Educational Psychology, 74 (4), p. 598-610.

Borko, H., Cone, R., Atwood-Russo, N., \& Shavelson, R. J. (1979). Teacher's decision making. Dans P. L. Peterson \& H. J. Walberg (Éd.), Reasearch on teaching. Concepts, findings and implications (p. 231-263). California: McCutchan Publishing Corporation.

Borko, H., \& Putnam, R. T. (1996). Learning to teach. Dans D. L. Berliner \& R. C. Calfee (Éd.), Handbook of educational psychology (p. 673-708). New York: MacMillan.

Borko, H., \& Shavelson, R. J. (1978). Teachers'sensitivity to the reliability of information in making causal attributions in an achievement situation. Journal of Educational Psychology, 70 (3), p. 271-279.

Borko, H., \& Shavelson, R. J. (1990). Teacher decision making. Dans B. F. Jones \& L. Idol (Éd.), Dimensions of thinking and cognitive instruction (p. 311-346). New Jersey: Erlbaum.

Bressoux, P. (2002). Les jugements des enseignants sur la valeur scolaire des élèves. Les stratégies de l'enseignant en situation d'interaction (p. 47-74). Retrouvé de http://archive-edutice.ccsd.cnrs.fr/docs/00/00/17/90/PDF/ Bressoux.pdf

Bressoux, P., \& Pansu, P. (1998). Norme d'internalité et activités évaluatives en milieu scolaire. Revue Française de Pédagogie, 122, p. 9-29.

Bromme, R. (1987). Teachers'assessment of students'difficulties and progress in understanding in the classroom. Dans J. Calderhead (Éd.), Exploring teachers'thinking (p. 125-146). London: Cassell.

Bromme, R. (1989). The « collective student » as the cognitive reference point of teachers'thinking about their students in the classroom. Dans J. Lowyck \& C. M. Clark (Éd.), Teacher thinking and professional action: Studia paedagogica $n^{\circ} 9$ (p. 209-222). Leuven, Belgium: Leuven University Press.

Bromme, R. (2005). The " collective student » as the cognitive reference point of teachers'thinking about their students in the classroom. Dans P. M. Denicolo \& M. Kompf (Éd.), Teacher thinking and professionnal action (p. 31-40). London: Routledge.

Brophy, J. E., \& McCaslin, M. (1992). Teachers'reports of how they perceive and cope with problem students. Elementary School Journal, 93, p. 3-68.

Buchmann, M. (1986). Role over person: legitimacy and authenticity in teaching. Dans M. Ben Peretz, R. Bromme, \& R. Halkes (Éd.), Advances of research on teacher thinking (p. 55-69). Lisse: Swets \& Zeitlinger B.V.

Buchmann, M. (1987). Role over person: justifying teacher action and decisions. Scandinavian Journal of Educational Research, 31, 1-21. 
Burns, R. (1987). Steering group, leveling effects, and instructional pace. American Journal of Education, 96, p. 24-55.

Cadwell, J., \& Jenkins, J. (1986). Teachers'judgments about their students: the effect of cognitive simplification on the rating process. American Educational Research Journal, 23 (3), p. 460-475.

Calderhead, J. (1996). Teachers: Beliefs and knowledge. Dans D. L. Berliner \& R. C. Calfee (Éd.), Handbook of educational psychology (p. 673-708). New York: MacMillan.

Chazan, D. (2000). Beyond formulas in mathematics and teaching: the dynamics of the high school algebra classroom. New York: Teachers College Press.

Chazan, D., \& Ball, D. (1999). Beyond being told not to tell. For the Learning of Mathematics, 19 (2), p. 2-10.

Clark, C. M., \& Peterson, P. L. (1986). Teachers'thought processes. Dans M. C. Wittrock (Éd.), Handbook of research on teaching (3 éd., p. 255-296). New York: Mac Millan.

Clark, C. M., \& Yinger, R. J. (1978). Research on teacher thinking (No. $\left.\mathrm{n}^{\circ} 12\right)$. Research Series. East Lansing: Michigan State University, Institute for Research on Teaching.

Clark, C. M., \& Yinger, R. J. (1979). Teachers'thinking. Dans P. L. Peterson \& H. J. Walberg (Éd.), Reasearch on teaching. Concepts, findings and implications (p. 231-263). California: McCutchan Publishing Corporation.

Cohen, D. K. (1990). À revolution in one classroom: the case of Mrs. Oublier. Educational Evaluation and Policy Analysis, 12, p. 327-345.

Colker, L. (1982). Teachers'interactive thoughts about pupil cognition (Unpublished Doctoral Dissertation). University of Illinois at Urbana-Champaign.

Cone, R. (1978). Teachers'decisions in managing student behavior: A laboratory simulation of interactive decision-making by teachers. Dans Annual Meeting of the American Educational Research Association. Toronto, Canada.

Conners, R. D. (1978). An analysis of teacher thought processes, beliefs, and principles during instruction (Unpublished Doctoral Dissertation). University of Alberta, Edmonton, Canada.

Cooper, H. M., Burger, J. M., \& Seymour, G. E. (1979). Classroom context and student ability as influences on teacher perception of classroom control. American Educational Research Journal, 16 (2), p. 189-196.

Cornett, J. W. (1990). Teacher thinking about curriculum and instruction: a case study of a secondary social studies teacher. Theory and Research in Social Education, 18 (3), p. 248-273.

Crahay, M., Wanlin, P., Issaieva, E., \& Laduron, I. (2010). Fonctions, structuration et évolution des croyances (et connaissances) des enseignants. Revue Française de Pédagogie, 172.

Creemers, B. P. M., \& Westerhof, K. (1982). Routinization of instructive and management behavior of teachers. The Netherlands, Haren: Educational Research Institute in the North.

Dahllöf, U. S. (1967). Skoldifferentering och undervisningsförlopp. Komparativa måloch processanalyser av skolsystem I. Stockholm: Almqvist \& Wiksell. 


\section{LA PENSÉE DES ENSEIGNANTS PENDANT L'INTERACTION EN CLASSE}

\section{Philippe Wanlin \& Marcel Crahay}

Dahllöf, U. S. (1971). Ability grouping, content validity, and curriculum process analysis (Teachers College Press.). New York.

Dahllöf, U. S., \& Lundgren, U. P. (1970). Macro- and micro- approaches combined for curriculum process analysis: a Swedish educational field project (No. $\left.\mathrm{n}^{\circ} 10\right)$. Reports from the Institute of Education. Göteborg: University of Göteborg.

Davies, D. (2003). Pragmatism, pedagogy and philosophy: a model of thought and action in primary technology and sience teacher education. International Journal of Technology and Design Education, 13, p. 207-211.

Denicolo, P. M. (1996). Productively confronting dilemmas in educational practice and research. Dans M. Kompf, W. Bond, D. Dworet, \& R. Boak (Éd.), Changing research and practice: teachers'profesionalism, identities, and knowledge (p. 56-66). London: Falmer Press.

Doyle, W. (1979). Making managerial decisions in classrooms. Dans D. L. Duke (Éd.), Classroom management (p. 42-74). Chicago: Chicago University Press.

Doyle, W. (1983). Academic work. Review of Educational Research, 53, p. 159-199.

Doyle, W. (1986). Classroom organisation and management. Dans M. C. Wittrock (Éd.), Handbook of research on teaching (3 éd., p. 392-431). New York: MacMillan.

Dunkin, M. J. (1986). Concepts et modèles dans l'analyse des processus d'enseignement. Dans M. Crahay \& D. Lafontaine (Éd.), L'art et la science de l'enseignement (p. 39-81). Bruxelles: Labor.

Elbaz, F. (1981). The teacher's « practical knowledge »: report of a case study. Curriculum Inquiry, 11, p. 43-71.

Erickson, F. (1982). Classroom discourse as improvisation: relationships between academic task structure and social participation structure in lessons. Dans L. C. Wilkinson (Éd.), Communicating in the classroom (p. 153-179). New York: Academic Press.

Fenstermacher, G. (1994). The knower and the known: the nature of knowledge in research on teaching. Dans L. Darling-Hammond (Éd.), Review of research in education (Vol. 20, p. 1-54). Washington, DC: American Educational Research Association.

Fischler, G. (1994). Concerning the difference between intention and action: teachers'conceptions and actions in physics teaching. Dans I. Carlgren, G. Handal, \& S. Vaage (Éd.), Teachers'minds and actions: research on teachers'thinking and practice (p. 165-). London: Falmer Press.

Fogarty, J. L., Wang, M. C., \& Creek, R. (1982). À descriptive study of experienced and novice teachers'interactive instructional decision processes. Dans Annual Meeting of the American Educational Research Association. New York City.

Freeman, D. J. (1991). To make the tacite explicite: teacher education, emerging discourse, and conceptions of teaching. Teaching and Teacher Education, 7 (5/6), p. 439-454.

Freeman, D. J., \& Porter, A. C. (1989). Do textbooks dictate the content of mathematics instruction in elementary schools? American Educational Research Journal, 26 (3), p. 403-421. 
Gamoran, A. (1986). Instructional and institutional effects of ability grouping. Sociology of Education, 59, p. 185-198.

Gamoran, A., \& Dreeben, R. (1986). Coupling and control in educational organizations. Administrative Science Quarterly, 31, p. 612-632.

Gastager, A. (2003). Paradigmenvielfalt aus Sicht des Unterrichtenden. Lengerich: Pabst.

Gilly, M. (1992). L'élève vu par le maître: influences socio-normatives dans l'exercice du rôle professionnel. Dans J. De Ketele (Éd.), L'évaluation: approche descriptive ou prescriptive? (p. 71-90). Bruxelles: De Boeck.

Good, T., Grouws, D. A., \& Beckerman, T. M. (1978). Curriculum pacing: some empirical data in mathematics. Journal of Curriculum Studies, 10, p. 75-81.

Goodlad, K., \& Klein, M. F. (1970). Behind the classroom door. Worthington, Ohio: Charles A. Jones Publishing Co.

Halkes, R., \& Deijkers, R. (2003). Teachers'teaching criteria. Dans M. Kompf \& P. M. Denicolo (Éd.), Teacher Thinking twenty years on: Revisiting persisting problems and advances in education (p. 3-13). The Netherlands, Lisse: Swets \& Zeitlinger B.V.

Haritos, C. (2004). Understanding teaching through the minds of teacher candidates: a curious blend of realism and idealism. Teaching and Teacher Education, 20, p. 637-654.

Hashweh, M. Z. (2003). Teacher accommodative change. Teaching and Teacher Education, 19, 421-434.

Herbst, P. G. (2003). Using novel tasks in teaching mathematics: three tensions affecting the work of teacher. American Educational Research Journal, 40 (1), p. 197-238.

Hoetker, J., \& Ahlbrand, W. P. (1969). The persistence of the recitation. American Educational Research Journal, 6, 145-167.

Hofer, M. (1981). Schülergruppierungen in Urteil und Verhalten des Lehrers. Dans M. Hofer (Éd.), Informationsverarbeitung und Entscheidungsverhalten von Lehrern (p. 192-221). München: Urban \& Schwarzenberg.

Hofer, M. (1986). Forming judgements in the classroom: how do teachers develop expectations of their students' performances? Dans M. Ben Peretz, R. Bromme, \& R. Halkes (Éd.), (p. 113-121). Lisse: Swets \& Zeitlinger B.V.

Hofer, M. (1989). Goal dependant perception in relations between teachers and students. Dans J. Lowyck \& C. M. Clark (Éd.), Teacher thinking and professional action: Studia paedagogica $n^{\circ} 9$ (p. 223-232). Leuven, Belgium: Leuven University Press.

Hofer, M. (2003). Forming judgements in the classroom: how do teachers develop expectations of their pupils'performances? Dans M. Kompf \& P. M. Denicolo (Éd.), Teacher Thinking twenty years on: Revisiting persisting problems and advances in education (p. 189-196). Lisse: Swets \& Zeitlinger B.V.

Hofer, M. (2005). Goal-dependant perception in relation between teachers and students. Dans P. M. Denicolo $\&$ M. Kompf (Éd.), Teacher thinking and professionnal action (p. 121-128). New York: Routledge. 


\section{LA PENSÉE DES ENSEIGNANTS PENDANT L'INTERACTION EN CLASSE}

Philippe Wanlin E Marcel Crahay

Hofer, M., \& Dobrick, M. (1981). Naive Ursachenzuschreibungen und Lehrerverhalten. Dans M. Hofer (Éd.), Informationsverarbeitung und Entscheidungsverhalten von Lehrern (p. 110-158). München: Urban \& Schwarzenberg.

Hoge, R. D., \& Coladarci, T. (1989). Teacher-baser judgements of academic achievement: a review of literature. Review of Educational Research, 59 (3), p. 297-313.

Housner, L. D., \& Griffey, D. C. (1983). Teacher cognition: Differences in planning and interactive decision making between experienced and inexperienced teachers. Dans Annual Meeting of the American Educational Research Association. Montreal, Canada.

Huber, G., \& Roth, J. (1990). Teachers'classroom activities and certainty/uncertainty orientation. Dans C. W. Day, M. Pope, \& P. M. Denicolo (Éd.), Insights into teachers'thinking and practice (p. 119-132). London: Falmer Press.

Jackson, P. (1968). Life in classrooms. New York: Holt, Rinehart and Winston, Inc.

Jackson, P. (1977). The Way New teachers Think. Dans J. Glidewell (Éd.), The Social Context of Learning and Development (p. 19-50). New York: Gardner Press.

Jackson, P. (1986). The Practice of Teaching. New York: Teachers College Press.

Jiménez, R. T., \& Gersten, R. (1999). Lessons and dilemmas derived from the literacy instruction of two latina/o teachers. American Educational Research Journal, 36 (2), p. 265-301.

Joyce, B. R. (1978). Toward a theory of information processing in teaching. Educational Research Quarterly, 3 (4), p. 66-77.

Kagan, D. (1992). Implications of research on teacher beliefs. Educational Psychologist, 27, p. 65-90.

Kansanen, P., Tirri, K., Meri, M., Krokfors, L., Husu, J., \& Jyrhämä, R. (2000). Teachers‘ pedagogical thinking. New York: Peter Lang.

Kleven, T. A. (1991). Interactive teacher decision-making - still a basic skill? Scandinavian Journal of Educational Research, 35, p. 287-294.

Kounin, J. S. (1970a). Discipline and group management in classrooms. New York: Holt, Rinehart \& Winston.

Kounin, J. S. (1970b). Observing and delineating technique of managing behaviour in classrooms. Journal of Research and Development in Education, 4, p. 62-70.

Kounin, J. S., \& Gump, P. V. (1974). Signal system of lesson settings and the task related behaviour of preschool children. Journal of Educational Psychology, 66 (4), p. 554-562.

Lam, B., \& Kember, D. (2006). The relationship between conceptions of teaching and approaches to teaching. Teachers and Teaching: Theory and Practice, 12 (6), p. 693-713.

Lampert, M. (1985). How do teachers manage to teach? Perspectives on problems in practice. Harvard Educational Review, 55, p. 178-194. 
Lampert, M. (1986). Teachers'strategies for understanding and managing classroom dilemmas. Dans M. Ben Peretz, R. Bromme, \& R. Halkes (Éd.), Advances of research on teacher thinking (p. 70-83). Lisse: Swets \& Zeitlinger B.V.

Lampert, M. (2001). Teaching problems and the problems of teaching. New Haven, CT: Yale University Press.

Leinhardt, G. (1983). Routines in expert math teachers'thoughts and actions. Présenté au Annual meeting of the American Educational Research Association, Montreal, Canada.

Leinhardt, G., \& Greeno, J. G. (1986). The cognitive skill of teaching. Journal of Educational Psychology, 78, p. $75-95$.

Lemmon, E. J. (1962). Moral dilemmas. The Philosophical Review, 70, p. 139-158.

Leuchter, M. (2009). Die Rolle der Lehrperson bei der Aufgabenbearbeeitung: Unterrichtsbezogene Kognitionen von Lehrpersonen. Münster: Waxmann.

Lindqvist, P., \& Nordänger, U. (2006). Who dares to disconnect in the age of uncertainty? Teachers' recesses and ,off-the-clock' work. Teachers and Teaching: Theory and Practice, 12 (6), p. 623-638.

Lissmann, U. (1989). Analysing teachers'thoughts prior to student assessment. Dans J. Lowyck \& C. M. Clark (Éd.), Teacher thinking and professional action: Studia paedagogica $n^{\circ} 9$ (p. 247-265). Leuven, Belgium: Leuven University Press.

Lissmann, U. (2005). Analysing teachers'thoughts prior to student assessment. Dans P. M. Denicolo \& M. Kompf (Éd.), Teacher thinking and professionnal action (p. 139-152). London: Routledge.

Lowyck, J. (1980). À process analysis of teaching (No. Report No. 21). Leuven, Belgium: Katholieke Universiteit te Leuven, Departement Pedagogische Wetenschappen.

Lowyck, J. (2003a). Teacher thinking and teacher routines: a bifurcation? Dans M. Kompf \& P. M. Denicolo (Éd.), Teacher Thinking twenty years on: Revisiting persisting problems and advances in education (p. 101-110). Lisse: Swets \& Zeitlinger B.V.

Lowyck, J. (2003b). Post-interactive reflections of teachers: a critical appraisal. Dans M. Kompf \& P. M. Denicolo (Éd.), Teacher Thinking twenty years on: Revisiting persisting problems and advances in education (p. 295-306). Lisse: Swets \& Zeitlinger B.V.

Lujan, J. (1980). Teacher warmth and student effort for high and low status students. Stanford University.

Lundgren, U. P. (1972). Frame factors and the teaching process: A contribution to Curriculum theory and theory of teaching. Stockholm: Almqvist \& Wiksell.

Lundgren, U. P. (1973). Pedagogical frames and the teaching process. À report from an empirical curriculum project. Dans Annual Meeting of the American Educational Research Association. New Orleans, Louisiana.

Lundgren, U. P. (1977). Model Analysis of Pedagogical Processes. Lund: Liber Publishing Company.

Lundgren, U. P. (1987). The environment. Dans M. J. Dunkin (Éd.), The international encyclopedia of teaching and teacher education (p. 525-530). New York: Pergamon Press. 


\section{LA PENSÉE DES ENSEIGNANTS PENDANT L'INTERACTION EN CLASSE}

Philippe Wanlin E Marcel Crahay

MacKay, D. A., \& Marland, P. W. (1978). Thought processes of teachers. Dans Annual Meeting of the American Educational Research Association. Toronto, Canada.

Mahnaz, M. (1994). An experienced teachers'model of thinking and teaching: An ethnographic study on teacher cognition. Dans Annual Meeting of the American Educational Research Association. New Orleans, LA.

Marland, P. (1986). Models of teachers'interactive thinking. The Elementary School Journal, 87 (2), p. 209-226.

Marland, P. (1993). Teachers'knowledge of students: a significant domain of practical knowledge? Retrouvé de http://www.aare.edu.au/93pap/marlp93147.txt

Marland, P. W. (1977). À study of teachers'interactive thoughts (Unpublished Doctoral Dissertation). University of Alberta, Edmonton, Canada.

Marx, R. W., \& Peterson, P. L. (1981). The natur of teacher decision making. Dans B. R. Joyce, C. C. Brown, \& L. Peck (Éd.), Flexibility in teaching: An excusion into the natur of teaching and training. New York: Longman.

Mayer, D., \& Marland, P. (1997). Teachers'knowledge of students : a significant domain of practical knowledge? Asia-Pacific Journal of Teacher Education, 25 (1), p. 17-34.

McLeod, M. A. (1981). The identification of intended learning outcomes by early childhood teachers: An exploratory study. University of Alberta, Edmonton, Canada.

McNair, K. (1978). Capturing inflight decisions. Educational Research Quarterly, 3, p. 26-42.

Morine, G., \& Vallance, E. (1975). Spacial study B: A study of teacher and pupil perceptions of classroom interaction (No. Tech. Rep. No. 75-11-6). San Francisco: Far West Laboratory.

Morine-Dershimer, G. (1978a). Planning and classroom reality: An in-depth look. Educational Research Quarterly, 3, p. 83-99.

Morine-Dershimer, G. (1978b). The anatomy of teacher prediction. Educational Research Quarterly, 3 (4), p. 59-65.

Morine-Dershimer, G. (1978c). How teachers « see » their pupils. Educational Research Quarterly, 3 (4), p. 43-52.

Morine-Dershimer, G. (1983). Instructional strategy and the «creation » of classroom status. American Educational Research Journal, 20 (4), p. 645-661.

Morine-Dershimer, G., \& Reeve, P. T. (1994). Studying teachers'thinking about instruction: issues related to analysis of metaphoric language. Dans I. Carlgren, G. Handal, \& S. Vaage (Éd.), Teachers'minds and actions: research on teachers'thinking and practice (p. 150-164). London: Falmer Press.

Morine-Dershimer, G., \& Tenenberg, M. (1981). Participant perspectives of classroom discourse. Executive summary of final report. Syracuse, N.Y.: Syracuse University Division for Study of Teaching.

Munby, H. (1983). À qualitative study of teachers'beliefs and principles. Présenté au Annual meeting of the American Educational Research Association, Montreal, Canada. 
Munthe, E., \& Thuen, E. (2009). Lower secondary school teachers'judgements of pupils'problems. Teachers and Teaching: Theory and Practice, 15 (5), p. 563-578.

Natriello, G., \& Dornbusch, S. M. (1983). Bringing behavior back in: the effects of student characteristics and behavior on the classroom behavior of teachers. American Educational Research Journal, 20 (1), p. 29-43.

Nyroos, M. (2008). Where does time go ? Teaching and time use from the perspective of teachers. Teachers and Teaching: Theory and Practice, 14 (1), p. 17-33.

Oldenbürger, H. A. (1986). Does a tendency to group pupils on attributes exist in teachers'cognitions/ judgements? Dans M. Ben Peretz, R. Bromme, \& R. Halkes (Éd.), Advances of research on teacher thinking (p. 186-200). Lisse: Swets \& Zeitlinger B.V.

Pajak, E., \& Blase, J. J. (1989). The impact of teachers' personal lives on professional role enactment: a qualitative analysis. American Educational Research Journal, 26 (2), p. 283-310.

Pajares, M. F. (1992). Teachers'beliefs and educational research: cleaning up a messy construct. Review of Educational Research, 62 (3), p. 307-332.

Parker, W. C., \& Gehrke, N. J. (1984). À grounded theory study of teachers'decision making. Présenté au Annual meeting of the American Educational Research Association, New Orleans, LA.

Parker, W. C., \& Gehrke, N. J. (1986). Learning activities and teachers‘ decisionmaking: some grounded hypotheses. American Educational Research Journal, 23 (2), p. 227-242.

Peterson, P. L., \& Clark, C. M. (1978). Teachers'reports of their cognitive processes during teaching. American Educational Research Journal, 15 (4), 555-565.

Peterson, P. L., Marx, R. W., \& Clark, C. M. (1978). Teacher planning, teacher behavior, and student achievement. American Educational Research Journal, 15 (3), p. 417-432.

Popham, W. J. (2004). À game without winners. Educational Leadership, 62, p. 46-50.

Prosner, G. J. (1987). Pacing and sequencing. Dans M. J. Dunkin (Éd.), International encyclopedia of teaching and teacher education (p. 266-272). New York: Pergamon Press.

Pullis, M., \& Cadwell, J. (1982). The influence of children's temperament characteristics on teachers'decision strategies. American Educational Research Journal, 19 (2), p. 165-181.

Putnam, J., \& Duffy, G. (1984). A descriptive study of the preactive and interactive decision making of an expert classroom teacher (No. ${ }^{\circ}$ 148). Research Series. East Lansing: Michigan State University, Institute for Research on Teaching.

Putnam, R. T. (1987). Structuring and adjusting content for students: A study of live and stimulated tutoring of addition. American Educational Research Journal, 24 (1), p. 13-48.

Remillard, J. T. (2005). Examining key concepts in research on teachers'use of mathematics curricula. Review of Educational Research, 75 (2), p. 211-246. 


\section{LA PENSÉE DES ENSEIGNANTS PENDANT L'INTERACTION EN CLASSE}

\section{Philippe Wanlin \& Marcel Crahay}

Resnick, L. B. (1977). Assuming that everyone can learn everything, will some learn less? School Review, 85, p. $445-452$.

Rothstein, R. (2004). The achievement gap: A broader picture. Educational Leadership, 62, p. 40-45.

Rowan, B., \& Miracle, A. W. (1983). Systems of ability grouping and the stratification of achievement in elementary schools. Sociology of Education, 56 (3), p. 133-144.

Sardo-Brown, D. (1990). Experienced teachers'planning practices: a US survey. Journal of Education for Teaching, 16 (1), p. 57-71.

Sartre, J. (1964). L'existentialisme est un humanisme. Paris: Nagel.

Semmel, D. S. (1977). The effects of training on teacher decision making. Dans Annual Meeting of the American Educational Research Association. New York City.

Shavelson, R. J. (1973). What is the basic teaching skill? The Journal of Teacher Education, 24 (2), p. 144-151.

Shavelson, R. J. (1976). Teachers'decision making. Dans N. L. Gage (Éd.), The psychology of teaching methods. Yearbook of the National Society for the Study of Education (p. 372-414). Chicago: Chicago University Press.

Shavelson, R. J., Atwood, N. K., \& Borko, H. (1977). Experiments on some factors contributing to teachers'pedagogical decisions. Cambridge Journal of Education, 7, p. 51-70.

Shavelson, R. J., Cadwell, J., \& Izu, T. (1977). Teachers'sensitivity to the reliability of information in making pedagogical decisions. American Educational Research Journal, 14 (2), p. 83-97.

Shavelson, R. J., \& Stern, P. (1981). Research on teachers'pedagogical thoughts, judgments, decisions, and behavior. Review of Educational Research, 51 (4), p. 455-498.

Shroyer, J. C. (1981). Critical moments in the teaching of mathematics: What makes teaching difficult? (Unpublished Doctoral Dissertation). Michigan State University, East Lansing.

Shulman, L. S. (1987). Knowledge and teaching: foundations of the new reform. Harvard Educational Review, 57 (1), p. 1-22.

Sinatra, G. M., \& Kardash, C. M. (2004). Teacher candidates'epistemological beliefs, dispositions, and views on teaching as persuasion. Contemporary Educational Psychology, 29, 483-489.

Smith, L. M., \& Geoffrey, W. (1968). The complexities of an urban classroom: an analysis toward a general theory of teaching. New York: Holt, Rinehart \& Winston.

Snow, R. E. (1972). À model teacher training system: An overview. Research and Development Memorandum 92. Stanford, California: Stanford Center for Research and Development in Teaching.

Solomon, D., \& Kendal, A. J. (1977). Dimensions of children's classroom behavior, as perceived by teachers. American Educational Research Journal, 14 (4), p. 411-421.

Styron, W. (1979). Le choix de Sophie. Paris: Folio. 
Sutcliffe, J., \& Whitfield, R. (1979). Classroom-based teaching decisions. Dans J. Eggleston (Éd.), Teacher decision-making in the classroom: A collection of papers (p. 8-37). London: Routledge \& Kegan Paul.

Tabachnick, B. R., \& Zeichner, K. M. (1986). Teacher beliefs and classroom behaviors: some responses to inconsistency. Dans M. Ben Peretz, R. Bromme, \& R. Halkes (Éd.), Advances of research on teacher thinking (p. 84-95). Lisse: Swets \& Zeitlinger B.V.

Tabachnick, B. R., \& Zeichner, K. M. (2003). Teachers` beliefs and classroom behaviours: some teacher responses to inconsistency. Dans M. Kompf \& P. M. Denicolo (Éd.), Teacher Thinking twenty years on: Revisiting persisting problems and advances in education (p. 165-175). Lisse: Swets \& Zeitlinger B.V.

Talanquer, V., Tomanek, D., \& Novodvorsky, I. (2007). Revealing student teachers'thinking through dilemma analysis. Journal of Science Teacher Education, 18, p. 399-421.

Tochon, F. V. (1990). Novice/Expert teachers'time epistemology. Présenté au Annual Meeting of the American Educational Research Association, Boston.

Tochon, F. V. (1993a). L'enseignant(e) expert(e). Paris: Nathan Pédagogie.

Tochon, F. V. (1993b). Le fonctionnement « improvisationnel » de l'enseignant expert. Revue des Sciences de l'Education, 19, p. 437-461.

Tochon, F. V., \& Dionne, J. (1994). Discourse analysis and instructional flexibility: a pragmatic grammar. Manograph Series, 5, p. 65-87.

Tomanek, D. (1994). À case of dilemmas: Exploring my assumptions about teaching science. Science Education, 78, p. 399-414.

Torff, B. (2006). Expert teachers'beliefs about use of critical-thinking activities with high- and low-advantage learners. Teacher Education Quarterly, 33 (2), p. 37-52.

Tournaki, N. (2003). Effects of student characteristics on teachers'predictions of students success. Journal of Educational Research, 36 (5), p. 310-319.

Vagle, M. D. (2009). Locating and exploring teacher perception in the reflective thinking process. Teachers and Teaching: Theory and Practice, 15 (5), p. 579-600.

Verloop, N., Van Driel, J., \& Meijer, P. (2001). Teacher knowledge and the knowledge base of teaching. International Journal of Educational Research, 35, p. 441-461.

Wagner, A. C. (2003). Conflicts in consciousness: imperative cognitions can lead to knots in thinking. Dans M. Kompf \& P. M. Denicolo (Éd.), Teacher Thinking twenty years on: Revisiting persisting problems and advances in education (p. 197-208). Lisse: Swets \& Zeitlinger B.V.

Wanlin, P. (2009a). La pensée des enseignants lors de la planification de leur enseignement. Revue Française de Pédagogie, 166, p. 89-128.

Wanlin, P. (2009b). High or low performance pupils: who do steer the instructional rhythm? Présenté au EARLI biennial conference, Amsterdam: University of Amsterdam. 


\section{LA PENSÉE DES ENSEIGNANTS PENDANT L'INTERACTION EN CLASSE}

Philippe Wanlin \& Marcel Crahay

Wanlin, P. (2009c). Teachers'cognitive and behavioural management of pupils'heterogeneousness during classroom interaction. Présenté au ISATT annual conference, Rovaniemi: University of Lapland.

Wanlin, P. (2010a). Quels dilemmes les enseignants doivent-ils gérer? Présenté au colloque de l'AREF, Genève: Université de Genève.

Warner, D. R. (1987). An exploratory study to identify the distinctive features of experienced teachers'thinking about teaching. University of New England (Australia).

Weinert, F. E., Knopf, M., \& Storch, C. (1981). Erwartungsbildung bei Lehrern. Dans M. Hofer (Éd.), Informationsverarbeitung und Entscheidungsverhalten von Lehrern (p. 157-191). München: Urban \& Schwarzenberg.

Westbury, I. (1973). Conventional classrooms, open classrooms, and the technology of teaching. Journal of Curriculum Studies, 5, p. 99-121.

Westbury, I. (1979). Schooling as an agency of education: some implication for curriculum theory. Dans W. B. Dockrell \& D. Hamilton (Éd.), Rethinking educational research. London: Hodder and Stoughton.

Williams, B. (1965). La fortune morale. Paris: PUF.

Windschitl, M. (2002). Framing constructivism in practice as the negotiation of dilemmas: an analysis of the conceptual, pedagogical, cultural, and political challenges facing teachers. Review of Educational Research, $72(2), 131-175$.

Wodlinger, M. G. (1980). À study of teacher interactive decision making (Unpublished Doctoral Dissertation). University of Alberta, Edmonton, Canada.

Woolfolk Hoy, A., Davis, H., \& Pape, S. (2006). Teachers'knowledge and beliefs. Dans P. A. Alexander \& P. H. Winne (Éd.), Handbook of educational psychology (2 éd., p. 715-737). Mahwah, NJ: Lawrence Erlbaum.

Yildirim, A. (2003). Instructional planning in a centralized school system: lessons of a study among primary school teachers in Turkey. International Review of Education, 49 (5), p. 525-543.

Yinger, R. J. (1977). À study of teacher planning: Description and theory development using ethnographic and information processing methods (Unpublished Doctoral Dissertation). Michigan State University, East Lansing.

Yinger, R. J. (1979). Routines in teacher planning. Theory into Practice, 18, p. 163-169.

Yinger, R. J. (1980). À study of teacher planning. Elementary School Journal, 80 (3), p. 107-127.

Yinger, R. J. (1987). By the seat of your pants: An inquiry into improvisation and taching. Dans Annual Meeting of the American Educational Research Association. Washington, DC. 